\title{
Determinants, Finite-Difference Operators and Boundary Value Problems ${ }^{\star}$
}

\section{Robin Forman}

Department of Mathematics, Rice University, Houston, TX 77251, USA

Received October 10, 1991

\begin{abstract}
We relate the determinants of differential and difference operators to the boundary values of solutions of the operators. Previous proofs of related results have involved considering one-parameter families of such operators, showing the desired quantities are equal up to a constant, and then calculating the constant. We take a more direct approach. For a fixed operator, we prove immediately that the two sides of our formulas are equal by using the following simple observation
\end{abstract} (Proposition 1.3): Let $U \in S U(n, \mathbf{C})$. Write $U$ in block form

$$
U=\left(\begin{array}{ll}
u_{11} & u_{12} \\
u_{21} & u_{22}
\end{array}\right),
$$

where $u_{11}$ and $u_{22}$ are square matrices. Then

$$
\operatorname{det} u_{11}=\overline{\operatorname{det} u_{22}} \text {. }
$$

\section{Introduction}

Motivated by questions in quantum field theory, there has been much recent interest in the problem of calculating the determinant of differential operators (see, for example, [Ra] chapter III). Suppose $L$ is a positive elliptic differential operator acting on sections of a vector bundle over a compact manifold. Then $L$ has a discrete spectrum

$$
\lambda_{1} \leqq \lambda_{2} \leqq \cdots \rightarrow \infty .
$$

Various methods have been used to make sense of

$$
\operatorname{det} L “=\Pi \lambda_{i} " \text {. }
$$

Perhaps the most common method is the zeta-function regularization of Ray and Singer [R-S], in which one defines $\log \operatorname{det} L$ by analytically continuing the function

$$
\sum \lambda_{i}^{-s} \log \lambda_{i}
$$

^ Partially supported by an NSF postdoctoral fellowship 
from $\operatorname{Re}(s)$ large to $s=0$. There are few general methods for evaluating such determinants. In [Fo1] we investigated one method of studying operators on manifolds with boundary, based on a reduction of calculations to the boundary of $M$.

A precursor to this general theory was the following example. Consider the operators

$$
L_{1}=-\frac{d^{2}}{d x^{2}}+R_{1}(x), \quad L_{2}=-\frac{d^{2}}{d x^{2}}+R_{2}(x)
$$

acting on functions $f$ on the interval $[0,1]$ which satisfy $f(0)=f(1)=0$. The operator $L_{1} L_{2}^{-1}$ is of the form (Identity) $+T$ where $T$ is trace class, and hence $L_{1} L_{2}^{-1}$ has a well-defined determinant (that is, without any regularization, see $[\mathrm{G}-\mathrm{K}])$. In fact ([D-D], [L-S])

$$
\left.\operatorname{det} L_{1} L_{2}^{-1}\right|_{\{f \mid f(0)=f(1)=0\}}=\frac{y_{1}(1)}{y_{2}(1)}
$$

where, for $i=1,2, y_{i}(x)$ is the unique solution to

$$
L_{i} y_{i}=0
$$

satisfying

$$
y_{i}(0)=0, \quad y_{i}^{\prime}(0)=1 .
$$

Note that $y_{i}(1)$ "lives" on the boundary of $[0,1]$.

Once we know that, defining det $L_{i}$ via zeta-function regularization,

$$
\frac{\operatorname{det} L_{1}}{\operatorname{det} L_{2}}=\operatorname{det}\left(L_{1} L_{2}^{-1}\right)
$$

([Fo1] Corollary 1.2) we can then reformulate $(0.1)$ as

$$
\left.\operatorname{det}\left(-\frac{d^{2}}{d x^{2}}+R(x)\right)\right|_{\{f \mid f(0)=f(1)=0\}}=c y(1),
$$

where $y$ is the unique solution of

$$
\begin{gathered}
\left(-\frac{d^{2}}{d x^{2}}+R(x)\right) y(x)=0, \\
y^{\prime}(0)=0, \quad y^{\prime}(0)=1,
\end{gathered}
$$

and $c$ is a constant independent of $R(x)$.

Another example of the general theory in [Fo1], also considered in [B-F-K], is the example of the above operator $L=-\frac{d^{2}}{d x^{2}}+R(x)$ acting on functions on $S^{1}$. This problem can be identified with $L$ acting on functions $f$ on $[0,1]$ satisfying

$$
f(0)=f(1), \quad f^{\prime}(0)=f^{\prime}(1) .
$$

We then have

$$
\left.\operatorname{det} L\right|_{\left\{f \mid f(0)=f(1), f^{\prime}(0)=f^{\prime}(1)\right\}}=c \operatorname{det}\left[\left(\begin{array}{ll}
1 & 0 \\
0 & 1
\end{array}\right)-\left(\begin{array}{cc}
z(1) & y(1) \\
z^{\prime}(1) & y^{\prime}(1)
\end{array}\right)\right],
$$


where $y(x)$ and $z(x)$ satisfy

$$
\begin{aligned}
& L y=L z=0 \text {, } \\
& y(0)=0 \quad y^{\prime}(0)=1, \\
& z(0)=1 \quad z^{\prime}(0)=0,
\end{aligned}
$$

and $c$ is independent of $R$. (The constant $c$ is studied in [B-F-K]).

What these formulae have in common is that the determinant of $L$, an operator acting on an $\infty$ dimensional space of functions is equated with the determinant of a finite dimensional $(1 \times 1$ or $2 \times 2)$ matrix whose entries are constructed from the boundary values of functions in the kernel of $L$. This may appear somewhat mysterious. The existing proofs offer little insight.

One type of proof, seen in [D-D] and [B-F-K], involves varying the operator analytically in a parameter $z$ and observing that both sides of $(0.1)$ are analytic in $z$ with the same zeros. Moreover, they have exponential growth order less than 1 . Thus, their ratio must be constant. This method exhibits a few disadvantages. First, although it is clear that the two sides of $(0.1)$ are zero at the same values of $z$, the proof that the zeros have the same order is surprisingly difficult (although a somewhat simpler topological proof is offered in [Fo2] Lemma 1.2). Second, the method gives no information about the constant of proportionality.

Another method, seen in [L-S] and [Fo1], involves proving that the two sides of $(0.1)$ have the same logarithmic derivative. This involves using detailed information about the resolvents of the differential operators. This method has the advantage of being better suited to generalization ([Fo1]), but again gives no information about the constant of proportionality.

The goal of this paper is to "demystify" these results by presenting greatly simplified proofs. The simplifications occur in two ways. First, we "generalize" the above results to operators on finite dimensional spaces (formula (0.1) is then derived by replacing the differential operators $L_{i}$ by suitable finite dimensional approximations). Second, the proof uses no analysis, neither analytic functions nor resolvents. Instead, we reduce the theorem to the following simple fact.

Proposition 1.3. Let $U \in S U(n, \mathbf{C})$. Write $U$ in block form

$$
U=\left(\begin{array}{ll}
u_{11} & u_{12} \\
u_{21} & u_{22}
\end{array}\right),
$$

where $u_{11}$ and $u_{22}$ are square matrices. Then

$$
\operatorname{det} u_{11}=\overline{\operatorname{det} u_{22}} \text {. }
$$

Note that this proposition does indeed relate the determinant of an operator on one space to the determinant of an operator on another space. Using Proposition 1.3 we prove directly that the two sides of $(0.1)$ are equal. That is, unlike earlier proofs, we do not vary the two sides at all. This new proof has the benefit of yielding immediately, with no extra effort, the precise value of the constant of proportionality.

Our general setting is the following. Suppose $V$ is a finite dimensional vector space equipped with an inner product, and

$$
L: V \rightarrow V
$$


is a linear map. For $A \subset V$ satisfying

$$
\operatorname{dim} A=\operatorname{dim} V-\operatorname{dim}(\operatorname{kernel} L)
$$

we consider

$$
L_{A}=\pi_{A} L \pi_{A}: A \rightarrow A,
$$

where $\pi_{A}$ denotes orthogonal projection onto $A$. Our main object of study is $\operatorname{det} L_{A}$. In this vein, we factor $L_{A}$ into simpler operators. Suppose $C$ is any space transverse to kernel $L$. Then we can write

$$
L_{A}=A \stackrel{\rho_{C}^{K}}{\longrightarrow} C \stackrel{L^{\longrightarrow}}{\longrightarrow} \operatorname{Image}(L) \stackrel{\pi_{A}}{\longrightarrow} A,
$$

where $\rho_{C}^{K}$ is the projection onto $C$ determined by the decomposition

$$
V=\operatorname{kernel} L+C \text {. }
$$

Thus

$$
\operatorname{det} L_{A}=\left(\operatorname{det} \rho_{C}^{K}: A \rightarrow C\right)(\operatorname{det} L: C \rightarrow \operatorname{Image}(L))\left(\operatorname{det} \pi_{A}: \operatorname{Image}(L) \rightarrow A\right) .
$$

The right-hand side is expressed in terms of determinants of operators taking one space to another. To make sense of these determinants we must choose volume forms for these spaces. (This is explained more precisely in Sect. 1). The term

$$
\operatorname{det} \pi_{A}: \text { Image }(L) \rightarrow A
$$

is simple to compute. In addition, we can choose $C$ so that

$$
\operatorname{det} L: C \rightarrow \operatorname{Image}(L)
$$

can be identified. We then apply Proposition 1.3 to prove

$$
\left(\operatorname{det} A \stackrel{\rho_{C}^{K}}{\longrightarrow} C\right)=\left(\operatorname{det} C^{\perp} \stackrel{\rho_{K}^{C}}{\longrightarrow} \operatorname{kernel} L \stackrel{\pi_{A^{\perp}}}{\longrightarrow} A^{\perp}\right),
$$

where $\rho_{K}^{C}$ denotes the projection onto kernel $L$ induced by (0.2). Combining (0.3), (0.4) and (0.5), evaluating $\operatorname{det} L_{A}$ is reduced to calculating the determinant of a map which factors through kernel $L$, a space which in our examples has dimension 1 or 2 .

Most of the results in the above mentioned papers follow immediately from this formula. To indicate how this works, in Sect. 2 we present some examples. We begin in part I with the simple application of the Laplacian $L$ on a graph $G$. That is

$$
V=\{f:(\text { Nodes of } G) \rightarrow \mathbf{C}\}
$$

and

$$
L: V \rightarrow V \text {. }
$$

Furthermore, $L$ is hermitian and the kernel of $L$ consists of the constant functions. Since

$$
\operatorname{dim} \operatorname{kernel} L=1
$$


the formula (0.5) is particularly easy to apply. A beautiful theorem of Kirchhoff [Ki] (see also [B-D], [Fo2] and [Fr]) states

$$
\operatorname{det} L_{(\operatorname{kernel} L)^{\perp}}\left[=\prod_{\substack{\lambda \in \operatorname{spec}(L) \\ \lambda \neq 0}} \lambda\right]=\#(\text { Nodes of } G) \#(\text { Spanning Trees in } G) .
$$

If

$$
M \subseteq N=\{\text { Nodes of } G\}
$$

is any subset, we define

$$
A_{M}=\left\{f \in V \mid \sum_{n \in M} f(n)=0\right\}
$$

(so that $A_{N}=(\operatorname{kernel} L)^{\perp}$ ). Then $(0.5)$ easily generalizes (0.6) to yield

$$
\operatorname{det} L_{A_{M}}=\# M \cdot \#(\text { Spanning Trees in } G) \text {. }
$$

In part II we consider general finite difference operators on an interval. As an example, let

$$
\tilde{L}=-\frac{d^{2}}{d x^{2}}+\tilde{R}(x)
$$

Divide the interval $[0,1]$ into $d$ equal segments and approximate $\tilde{L}$ by the standard finite difference approximation

$$
L=-\nabla^{2}+R: V \rightarrow V,
$$

where

$$
V=\left\{f:\left\{0, \frac{1}{d}, \ldots, 1\right\} \rightarrow \mathbf{C}\right\}
$$

and $\nabla$ denotes the finite difference approximation for $d / d x$. We then restrict $L$ to various subspaces of $V$ (corresponding to boundary conditions). Three special cases of the general boundary conditions considered in this section are:

Dirichlet Boundary conditions $=A_{\infty}=\{f \in V \mid f(0)=f(1)=0\}$,

Neumann Boundary Conditions $=A_{0}=\left\{f \in V \mid f(0)=f\left(\frac{1}{d}\right), f\left(\frac{d-1}{d}\right)=f(1)\right\}$,

Periodic Boundary Conditions $=B_{1}=\left\{f \in V \mid f(0)=f\left(\frac{d-1}{d}\right), f\left(\frac{1}{d}\right)=f(1)\right\}$.

Formula (0.5) yields

Theorem 2.2. $\operatorname{det} L_{A_{\infty}}=d^{2 d-1} y(1)$, where $y$ is the unique element in kernel $L$ satisfying

$$
y(0)=0, \quad \nabla y(0)=1 .
$$

Corollary 2.5. $\operatorname{det} L_{A_{0}}=\frac{d^{2 d-3}}{4} \nabla z\left(\frac{d-1}{d}\right)$, where $z \in \operatorname{kernel} L$ satisfies

$$
z(0)=1, \quad \nabla z(0)=0 .
$$


Theorem 2.6.

$$
\operatorname{det} L_{B_{1}}=\frac{-d^{2 d-2}}{4} \operatorname{det}\left[\left(\begin{array}{ll}
1 & 0 \\
0 & 1
\end{array}\right)-\left(\begin{array}{cc}
z\left(\frac{d-1}{d}\right) & y\left(\frac{d-1}{d}\right) \\
\nabla z\left(\frac{d-1}{d}\right) & \nabla y\left(\frac{d-1}{d}\right)
\end{array}\right)\right]
$$

Note that in each case the left-hand side is the determinant of an operator on a $d-1$ dimensional space, since

$$
\operatorname{dim} A_{\infty}=\operatorname{dim} A_{0}=\operatorname{dim} B_{1}=\operatorname{dim}(\operatorname{kernel} L)^{\perp}=d-1 .
$$

As our approximations become finer, that is as $d \rightarrow \infty$, this dimension goes to $\infty$. On the other hand, the right-hand side does not become more complicated as $d \rightarrow \infty$. This is due to the fact that

$$
\operatorname{dim} A_{\infty}^{\perp}=\operatorname{dim} A_{0}^{\perp}=\operatorname{dim} B_{1}^{\perp}=\operatorname{dim} k e r n e l ~ L=2
$$

does not grow with $d$. Note also that there are no unknown constants.

We investigate the effect of adding a first order term to the operator $L$ in Sect. 2(II), observation 4.

In Sect. 3 we provide the analysis which allows us to deduce formula (0.1) for differential operators from the corresponding formula for finite difference operators. Unlike Sects. 1 and 2, this section is very technical. The reader willing to believe some technical results may skip this section without detriment.

The results in this paper may have relevance for the attempts to study quantum field theory via finite dimensional approximations.

\section{The Main Theorem}

Let $V$ be a finite dimensional vector space defined over $\mathbf{R}$ or $\mathbf{C}$, with a (Euclidean or Hermitian, respectively) inner product, which we denote by $\langle$, $\rangle$. Let

$$
L: V \rightarrow V
$$

be any hermitian map (this restriction will be removed later). Then for any subspace

$$
A \subset V
$$

we define

$$
L_{A}=\pi_{A} L \pi_{A}: A \rightarrow A,
$$

where $\pi_{A}$ denotes the orthogonal projection onto $A$. Our goal is to study $\operatorname{det} L_{A}$.

As an example, let $K$ denote the kernel of $L$, and $K^{\perp}$ the orthogonal complement. Then

$$
\operatorname{det} L_{K^{\perp}}=\prod_{\substack{\lambda \in \operatorname{spec}(L) \\ \lambda \neq 0}} \lambda,
$$

where $\operatorname{spec}(L)$ denotes the set of eigenvalues of $L$ taken with their appropriate multiplicity. 
In fact, for any $A, L_{A}$ factors through $L_{K^{\perp}}$ :

$$
L_{A}: A \stackrel{\pi_{K^{\perp}}}{\longrightarrow} K^{\perp} \stackrel{L_{K^{\perp}}}{\longrightarrow} K^{\perp} \stackrel{\pi_{A}}{\longrightarrow} A
$$

Thus, it follows that if $\operatorname{dim} A=\operatorname{dim} K^{\perp}$,

$$
\frac{\operatorname{det} L_{A}}{\operatorname{det} L_{K^{\perp}}}=\operatorname{det} \pi_{A} \pi_{K^{\perp}}: A \rightarrow K^{\perp} \rightarrow A .
$$

Note that the right-hand side depends only on the metric and the spaces $A$ and $K^{\perp}$.

It is convenient at this point to be able to speak of the determinant of a map between two different spaces. Suppose $X$ and $Y$ are two spaces of the same dimension, each equipped with an inner product. Then, for any linear map

$$
H: X \rightarrow Y
$$

we cannot define det $H$, but we set

$$
|\operatorname{det} H|=\left(\operatorname{det} H^{*} H: X \rightarrow X\right)^{\frac{1}{2}},
$$

where $H^{*}$ denotes the adjoint of $H$ with respect to the inner products. The following 2 properties are immediate:

(i) $|\operatorname{det} H|=\left|\operatorname{det} H^{*}\right|$,

(ii) Suppose $X, Y, Z$ are 3 spaces of the same dimension, each equipped with an inner product. Then, for any maps

$$
\begin{aligned}
& H_{1}: X \rightarrow Y \\
& H_{2}: Y \rightarrow Z
\end{aligned}
$$

we have

$$
\left|\operatorname{det} H_{2} \circ H_{1}\right|=\left|\operatorname{det} H_{2}\right|\left|\operatorname{det} H_{1}\right| \text {. }
$$

We note that for any two subspaces $X$ and $Y$ of $V$, the adjoint of the map

$$
\pi_{Y}: X \rightarrow Y
$$

is

$$
\pi_{X}: Y \rightarrow X
$$

Thus, if

$$
\operatorname{dim} X=\operatorname{dim} Y
$$

we have

$$
\left|\operatorname{det} \pi_{Y}: X \rightarrow Y\right|=\left(\operatorname{det} \pi_{X} \pi_{Y}: X \rightarrow Y \rightarrow X\right)^{\frac{1}{2}} .
$$

Together, (1.1) and (1.6) yield

Theorem 1.1. If

$$
\operatorname{dim} A=\operatorname{dim} K^{\perp}
$$

then

$$
\frac{\operatorname{det} L_{A}}{\operatorname{det} L_{K^{\perp}}}=\left|\operatorname{det} \pi_{K^{\perp}}: A \rightarrow K^{\perp}\right|^{2}=\left|\operatorname{det} \pi_{A}: K^{\perp} \rightarrow A\right|^{2}
$$


In examples, $K=\operatorname{kernel}(L)$ tends to be much smaller than $K^{\perp}$. Thus, our next goal is to restate the right-hand side of (1.7) in terms of $K$, rather than $K^{\perp}$.

Before proceeding, for future reference it will be useful to broaden our perspective. Suppose

$$
H: X \rightarrow Y
$$

is linear, where $X$ and $Y$ are two spaces of the same dimension, each equipped with an inner product. Rather than dealing only with

$$
|\operatorname{det} H| \in \mathbf{R}^{+} \text {, }
$$

if we have volume forms for $X$ and $Y$ we can define a value for

$$
\operatorname{det} H \in \mathbf{C} \text {. }
$$

That is, suppose

$$
\begin{aligned}
& \mathscr{X}=x_{1} \wedge \cdots \wedge x_{n} \subset \Lambda^{n} X, \\
& \mathscr{Y}=y_{1} \wedge \cdots \wedge y_{n} \subset \Lambda^{n} Y
\end{aligned}
$$

( $n=\operatorname{dim} X=\operatorname{dim} Y$ ) are unit length volume forms (for example, if $\left\{x_{i}\right\}$ and $\left\{y_{i}\right\}$ are orthonormal bases). Then we can define $\operatorname{det} H$ with respect to $\mathscr{X}$ and $\mathscr{Y}$ by

$$
H x_{1} \wedge \cdots \wedge H x_{n}=(\operatorname{det} H) \mathscr{Y} .
$$

The forms $\mathscr{X}$ and $\mathscr{Y}$ are determined up to multiplication by complex numbers of norm 1 . Varying our choice of $\mathscr{X}$ and $\mathscr{Y}$ may vary the phase of det $H$ (although (1.9) gives a well-defined value for $|\operatorname{det} H|$ which, as we will see below, agrees with our previous definition). Defining all determinants with respect to fixed volume forms $\mathscr{X}$ and $\mathscr{Y}$ we have the following refinements of (1.3) and (1.4).

(i) $\operatorname{det} H=\overline{\operatorname{det} H^{*}}$.

(Note that this implies that the definition of $\operatorname{det} H$ is compatible with our previous definition (1.2) of $|\operatorname{det} H|)$.

Proof. If $\mathscr{X}$ and $\mathscr{Y}$ have unit length, then $\mathscr{X}$ and $\mathscr{Y}$ are of the form (1.8) for some orthonormal bases $\left\{x_{i}\right\}$ and $\left\{y_{i}\right\}$. Define an $n \times n$ matrix $\mathscr{H}$ by

$$
\mathscr{H}_{i j}=\left\langle H x_{i}, y_{j}\right\rangle \text {. }
$$

Then, it follows immediately from (1.9) that

$$
\operatorname{det} H=\operatorname{det} \mathscr{H} \text {. }
$$

Furthermore

$$
\left\langle H^{*} y_{i}, x_{j}\right\rangle=\left\langle y_{i}, H x_{j}\right\rangle=\overline{\mathscr{H}_{j i}}=\left(\mathscr{H}^{*}\right)_{i j}
$$

so that

$$
\operatorname{det} H^{*}=\operatorname{det} \mathscr{H}^{*}=\overline{\operatorname{det} \mathscr{H}}=\overline{\operatorname{det} H} \text {. }
$$

(ii) Suppose $X, Y$ and $Z$ are three spaces of the same dimension with volume forms $\mathscr{X}, \mathscr{Y}$ and $\mathscr{Z}$, respectively. If

$$
H_{1}: X \rightarrow Y, \quad H_{2}: Y \rightarrow Z
$$


are linear, then, defining all determinants with respect to $\mathscr{X}, \mathscr{Y}$ and $\mathscr{Z}$, we have

$$
\operatorname{det} H_{2} \circ H_{1}=\operatorname{det} H_{2} \operatorname{det} H_{1} \text {. }
$$

The main tool in all of our future work is the following lemma, which will enable us to relate the determinants of operators between different pairs of spaces.

Lemma 1.2. Let

$$
V=X+X^{\perp}=Y+Y^{\perp}
$$

be two orthogonal decompositions satisfying

$$
\operatorname{dim} X=\operatorname{dim} Y \text {. }
$$

Suppose further that $X, X^{\perp}, Y$ and $Y^{\perp}$ are equipped with unit length volume forms $\mathscr{X}$, $\mathscr{X}^{\perp}, \mathscr{Y}$ and $\mathscr{Y}^{\perp}$, respectively, which satisfy

$$
\mathscr{X} \wedge \mathscr{X}^{\perp}=\mathscr{Y} \wedge \mathscr{Y}^{\perp} .
$$

Then, defining all determinants with respect to $\mathscr{X}, \mathscr{X}^{\perp}, \mathscr{Y}$ and $\mathscr{Y}^{\perp}$

$$
\operatorname{det} \pi_{Y}: X \rightarrow Y=\operatorname{det} \pi_{X^{\perp}}: Y^{\perp} \rightarrow X^{\perp} .
$$

(From (1.5) and (1.10), the right-hand side is equal to

$$
\overline{\left.\operatorname{det} \pi_{Y^{\perp}}: X^{\perp} \rightarrow Y^{\perp}\right)} \text {. }
$$

We will reduce this lemma to a statement about special unitary matrices.

Let

$$
\left\{x_{1}, \ldots, x_{k}\right\}, \quad\left\{x_{1}^{\perp}, \ldots, x_{l}^{\perp}\right\}, \quad\left\{y_{1}, \ldots, y_{k}\right\}, \quad\left\{y_{1}^{\perp}, \ldots, y_{l}^{\perp}\right\}
$$

be orthonormal bases of $X, X^{\perp}, Y$ and $Y^{\perp}$, respectively. Then

$$
\left\{x_{1}, \ldots, x_{k}, x_{1}^{\perp}, \ldots, x_{l}^{\perp}\right\},\left\{y_{1}, \ldots, y_{k}, y_{1}^{\perp}, \ldots, y_{l}^{\perp}\right\}
$$

are orthonormal bases of $V$. Let $U$ denote the matrix corresponding to the change of basis

$$
\left\{x_{1}, \ldots, x_{l}^{\perp}\right\} \rightarrow\left\{y_{1}, \ldots, y_{l}^{\perp}\right\}
$$

That is

$$
U_{i j}= \begin{cases}\left\langle x_{i}, y_{j}\right\rangle & 1 \leqq i, j \leqq k \\ \left\langle x_{i}, y_{j-k}^{\perp}\right\rangle & 1 \leqq i \leqq k, k+1 \leqq j \leqq k+l \\ \left\langle x_{i-k}^{\perp}, y_{j}\right\rangle & k+1 \leqq j \leqq k+l, 1 \leqq j \leqq k \\ \left\langle x_{i-k}^{\perp}, y_{j-k}^{\perp}\right\rangle & k+1 \leqq i, j \leqq k+l .\end{cases}
$$

Then, from (1.12), $U$ is unitary. From (1.11), $\operatorname{det} U=1$, so that

$$
U \in S U(k+l, \mathbf{C}) \text {. }
$$

Write $U$ in the block form

$$
\left(\begin{array}{ll}
u_{11} & u_{12} \\
u_{21} & u_{22}
\end{array}\right)
$$


where

$$
\begin{array}{ll}
u_{11}=\left(U_{i j}\right)_{1 \leqq i, j \leqq k} & u_{12}=\left(U_{i j}\right)_{i \leqq k, j \geqq k+1} \\
u_{21}=\left(U_{i j}\right)_{i} \geqq k+1, j \leqq k & u_{22}=\left(U_{i j}\right)_{k+1 \leqq i, j \leqq k+l}
\end{array}
$$

Then

$$
\begin{gathered}
\operatorname{det} \pi_{Y}: X \rightarrow Y=\operatorname{det} u_{11} \\
\operatorname{det} \pi_{Y^{\perp}}: X^{\perp} \rightarrow Y^{\perp}=\operatorname{det} u_{22} .
\end{gathered}
$$

Thus, the lemma follows from the following proposition

Proposition 1.3. Let $U \in S U(n, \mathbf{C})$ be expressed in block form

$$
U=\left(\begin{array}{ll}
u_{11} & u_{12} \\
u_{21} & u_{22}
\end{array}\right)
$$

so that $u_{11}$ and $u_{22}$ are square matrices. Then

$$
\operatorname{det} u_{11}=\overline{\operatorname{det} u_{22}} \text {. }
$$

Proof. Since $U \in S U(n, \mathbf{C})$ we have

$$
U U^{*}=\left(\begin{array}{ll}
u_{11} & u_{12} \\
u_{21} & u_{22}
\end{array}\right)\left(\begin{array}{ll}
u_{11}^{*} & u_{21}^{*} \\
u_{12}^{*} & u_{22}^{*}
\end{array}\right)=\left(\begin{array}{ll}
1 & 0 \\
0 & 1
\end{array}\right) .
$$

Thus, using the second column of (1.14), we find

$$
\left(\begin{array}{ll}
u_{11} & u_{12} \\
u_{21} & u_{22}
\end{array}\right)\left(\begin{array}{ll}
1 & u_{21}^{*} \\
0 & u_{22}^{*}
\end{array}\right)=\left(\begin{array}{ll}
u_{11} & 0 \\
u_{21} & 1
\end{array}\right)
$$

Taking determinants yields

$$
\operatorname{det} u_{22}^{*}=\operatorname{det} u_{11}
$$

as desired.

Substituting Lemma 1.2 into Theorem 1.1 yields

Corollary 1.4. (i) If

$$
\operatorname{dim} A=\operatorname{dim} K^{\perp} .
$$

Then

(ii) If

$$
\frac{\operatorname{det} L_{A}}{\operatorname{det} L_{K^{\perp}}}=\left|\operatorname{det} \pi_{A^{\perp}}: K \rightarrow A^{\perp}\right|^{2}
$$

$$
\operatorname{dim} A=\operatorname{dim} B=\operatorname{dim} K^{\perp}
$$

and

$$
\operatorname{det} L_{B} \neq 0
$$

then

$$
\frac{\operatorname{det} L_{A}}{\operatorname{det} L_{B}}=\frac{\left|\operatorname{det} \pi_{A^{\perp}}: K \rightarrow A^{\perp}\right|^{2}}{\left|\operatorname{det} \pi_{B^{\perp}}: K \rightarrow B^{\perp}\right|^{2}}=\left|\operatorname{det} \Phi_{A B}: B^{\perp} \rightarrow K \rightarrow A^{\perp}\right|^{2}
$$


where

$$
\Phi_{A B}=B^{\perp} \stackrel{\left(\pi_{B^{\perp}}\right)^{-1}}{\longrightarrow} K \stackrel{\pi_{A^{\perp}}}{\longrightarrow} A^{\perp}
$$

(This is a matrix version of Theorem 1 of [Fo1].)

The value of formula (1.15) largely rests on our ability to choose a space $B$ for which we can calculate det $L_{B}$. In what follows, it will help us greatly to be able to consider slightly more general operators for our denominator.

From this point on, we will no longer assume that

$$
L: V \rightarrow V
$$

is hermitian. Let $C \subset V$ be any linear subspace such that

$$
\operatorname{dim} C=\operatorname{dim} V-\operatorname{dim}(K=\operatorname{kernel}(L))
$$

and

$$
C \cap K=\{0\}
$$

Then we can write

$$
V=C+K,
$$

although this decomposition is not necessarily orthogonal. Let

$$
\rho_{C}^{K}: V \rightarrow C
$$

denote the projection onto $C$ induced by (1.18). The symbol $\pi$ will be reserved for orthogonal projections, so that, for example, for any space $A$

$$
\pi_{A}=\rho_{A}^{A^{\perp}} \text {. }
$$

Now, for any $v \in V$

$$
v=\rho_{C}^{K}(v)+k
$$

for some $k \in K$, so that

$$
L v=L \rho_{C}^{K}(v) .
$$

This implies that for every $A$ satisfying

$$
\operatorname{dim} A=\operatorname{dim} V-\operatorname{dim} K
$$

$L_{A}$ factors through $L \rho_{C}^{K}$. Namely

$$
L_{A}=A \stackrel{\rho_{C}^{K}}{\longrightarrow} C \stackrel{L}{\longrightarrow} \operatorname{Im}(L) \stackrel{\pi_{A}}{\longrightarrow} A
$$

(where $\operatorname{Im}(L)=\operatorname{Image}(L))$. Thus

$$
\operatorname{det} L_{A}=\left(\operatorname{det} \rho_{C}^{K}: A \rightarrow C\right)(\operatorname{det} L: C \rightarrow \operatorname{Im}(L))\left(\operatorname{det} \pi_{A}: \operatorname{Im}(L) \rightarrow A\right) .
$$

Our last simplification is to note that $\rho_{C}^{K}$ factors through $K^{\perp}$. Namely

$$
\rho_{C}^{K}: A \stackrel{\pi_{K^{\perp}}}{\longrightarrow} K^{\perp} \stackrel{\rho_{C}^{K}}{\longrightarrow} C
$$


so that

$$
\left(\operatorname{det} \rho_{C}^{K}: A \rightarrow C\right)=\left(\operatorname{det} \pi_{K^{\perp}}: A \rightarrow K^{\perp}\right)\left(\operatorname{det} \rho_{C}^{K}: K^{\perp} \rightarrow C\right) .
$$

From Lemma 1.2, with appropriately chosen volume forms

$$
\operatorname{det} \pi_{K^{\perp}}: A \rightarrow K^{\perp}=\operatorname{det} \pi_{A^{\perp}}: K \rightarrow A^{\perp} .
$$

Moreover

$$
K^{\perp} \stackrel{\rho_{C}^{K}}{\longrightarrow} C=K^{\perp} \stackrel{\left(\pi_{K^{\perp}}\right)^{-1}}{\longrightarrow} C
$$

so that

$$
\operatorname{det} \rho_{C}^{K}: K^{\perp} \rightarrow C=\left(\operatorname{det} \pi_{K^{\perp}}: C \rightarrow K^{\perp}\right)^{-1}=\left(\operatorname{det} \pi_{C^{\perp}}: K \rightarrow C^{\perp}\right)^{-1}
$$

by Corollary 1.4. Now, using that

$$
K \stackrel{\pi_{C^{\perp}}}{\longrightarrow} C^{\perp}=K \stackrel{\left(\rho_{K}^{c}\right)^{-1}}{\longrightarrow} C^{\perp}
$$

we find that (1.23) is equal to

$$
\operatorname{det} \rho_{K}^{C}: C^{\perp} \rightarrow K .
$$

Combining (1.21), (1.22) and (1.24) we see that

$$
\operatorname{det} \rho_{C}^{K}: A \rightarrow C=\operatorname{det} C^{\perp} \stackrel{\rho_{K}^{C}}{\longrightarrow} K \stackrel{\pi_{A^{\perp}}}{\longrightarrow} A^{\perp} .
$$

Substituting into (1.20) we find

Theorem 1.5. Let $C$ be any linear subspace of $V$ satisfying (1.16) and (1.17) and $A$ any subspace satisfying (1.19). Then, with respect to appropriate volume forms on $A, A^{\perp}$, $C, C^{\perp}$ and $\operatorname{Im}(L)$ we have

$$
\operatorname{det} L_{A}=\left(\operatorname{det} C^{\perp} \stackrel{\rho_{K}^{C}}{\longrightarrow} K \stackrel{\pi_{A^{\perp}}}{\longrightarrow} A\right)(\operatorname{det} L: C \rightarrow \operatorname{Im}(L))\left(\operatorname{det} \pi_{A}: \operatorname{Im}(L) \stackrel{\pi_{A}}{\longrightarrow} A\right) .
$$

The significance of Theorem 1.5 is demonstrated in Sect. 2(II), in which, for a large class of finite difference operators $L$, we find a space $C$ (in the examples $C=$ Cauchy data) such that

$$
\operatorname{det} L: C \rightarrow \operatorname{Im}(L)
$$

can be readily calculated. The space $\operatorname{Im}(L)$ is easily identifiable and hence

$$
\operatorname{det} \pi_{A}: \operatorname{Im}(L) \rightarrow A
$$

can be calculated. Thus, Theorem 1.5 relates $\operatorname{det} L_{A}$ to $\operatorname{det} \pi_{A^{\perp}} \rho_{K}^{C}: C^{\perp} \rightarrow A^{\perp}$. This has the primary advantage that $\operatorname{dim} A^{\perp}=\operatorname{dim} C^{\perp}$ is much smaller than $\operatorname{dim} A$. Moreover, we identify $\pi_{A^{\perp}} \rho_{K}^{C}$ : $C^{\perp} \rightarrow A^{\perp}$ with a classical analytic operator.

\section{Applications}

I. Laplacians on Graphs. Let $G$ be a connected graph consisting of a finite set of nodes $N$ and a finite set of edges $E$. We allow multiple edges between vertices. Let $V=N^{*}$ be the space of maps

$$
f: N \rightarrow \mathbf{C}
$$


Define a Laplacian

$$
L: V \rightarrow V
$$

by setting, for $f \in V, n \in N$

$$
(L f)(n)=d(n) f(n)-\sum_{e \in E(n)} f(\bar{e}),
$$

where $E(n)$ is the set of edges attached to $n$,

$$
d(n)=\# E(n)
$$

and, for $e \in E(n), \bar{e}$ denotes the other endpoint of $e$.

There is a canonical basis of $V$ given by $\left\{f_{n}\right\}_{n \in N}$, where, for $n, m \in N$

$$
f_{n}(m)=\left\{\begin{array}{lll}
1 & \text { if } & m=n \\
0 & \text { if } & m \neq n
\end{array} .\right.
$$

We define an inner product $\langle$,$\rangle on V$ by declaring $\left\{f_{n}\right\}$ to be an orthonormal basis. The Laplacian $L$ is hermitian with respect to $\langle$,$\rangle .$

It can be seen directly from (2.1) that the kernel of $L$ is 1-dimensional, consisting of the constant functions.

The operator $L$ plays a crucial role in electrical network theory. It is in this context that Krichhoff proved his beautiful theorem [Ki] (see also [B-D, Fr, and Fo3]) that

$$
\operatorname{det} L_{K^{\perp}}\left[=\prod_{\substack{\lambda \in \operatorname{spec}(L) \\ \lambda \neq 0}} \lambda\right]=\# N \cdot \#\{\text { maximal trees in } G\},
$$

where a tree is a subgraph of $G$ containing no cycles.

For any subset $M \subseteq N$ we define a space $A_{M} \subset V$ by

$$
A_{M}=\left\{f \in V \mid \sum_{m \in M} f(m)=0\right\} .
$$

Then, if $M=N$ we have $A_{M}=K^{\perp}$, where $K=\operatorname{kernel}(L)$. If $M=\{n\}$ then

$$
A_{M}=\{f \in V \mid f(n)=0\} .
$$

If $G$ represents an electrical circuit, then $A_{M}$ corresponds to the possible potentials on the vertices if $\{n\}$ is grounded.

Applying Corollary 1.4 we learn

Theorem 2.1. For any $M \subseteq N$

$$
\operatorname{det} L_{A_{M}}=\# M \cdot \#\{\text { maximal trees in } G\} .
$$

Proof. From Corollary 1.4 and (2.2)

$$
\operatorname{det} L_{A_{M}}=\# N \cdot \#\{\text { maximal trees in } G\}\left|\operatorname{det} \pi_{A_{M}}: K \rightarrow A_{M}^{\frac{1}{M}}\right|^{2} .
$$

An orthonormal basis for $K$ is given by $f_{K}$ where

$$
f_{K}(n)=\frac{1}{(\# N)^{\frac{1}{2}}} \text { for every } n \in N
$$


An orthonormal basis for $A_{M}^{\perp}$ is given by $f_{M}$ where

$$
f_{M}(n)=\left\{\begin{array}{cc}
0 & n \notin M \\
\frac{1}{(\# M)^{\frac{1}{2}}} & n \in M
\end{array}\right.
$$

Thus

$$
\pi_{A_{M}^{\perp}}\left(f_{K}\right)=\left\langle f_{K}, f_{M}\right\rangle f_{M}
$$

so that

$$
\begin{aligned}
\operatorname{det}\left(\pi_{A_{M}^{\frac{1}{M}}}: K \rightarrow A_{M}^{\perp}\right)=\left\langle f_{K}, f_{M}\right\rangle & =\sum_{n \in M} f_{K}(n) f_{M}(n) \\
& =\sum_{n \in M} \frac{1}{(\# N)^{\frac{1}{2}}(\# M)^{\frac{1}{2}}}=\frac{(\# M)^{\frac{1}{2}}}{(\# N)^{\frac{1}{2}}} .
\end{aligned}
$$

Substituting (2.4) into (2.3) yields Theorem 2.1.

II. Boundary Value Problems on an Interval. We approximate the unit interval by $d+1$ nodes

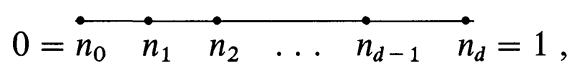

where $n_{i}=i / d$. Let

$$
N=\left\{n_{0}, \ldots, n_{d}\right\} .
$$

We approximate $\mathbf{C}^{r}$ valued functions on the interval by

$$
V=N^{*}=\left\{f: N \rightarrow \mathbf{C}^{r}\right\} \text {. }
$$

Let $L$ be a linear operator on $V$ of the form

$$
(L f)\left(n_{i}\right)=\left\{\begin{array}{ll}
\sum_{j=-a}^{b} c_{j}(i) f\left(n_{i+j}\right) & \text { if } a \leqq i \leqq d-b \\
0 & \text { if } i<a \text { or } i>d-b
\end{array},\right.
$$

where $a$ and $b$ are non-negative integers, not both zero, and each $c_{j}(i)$ is an $r \times r$ matrix. $L$ is a finite difference approximation of a differential operator of order $a+b$. We require $L$ to be elliptic. This is reflected in the condition

$$
\operatorname{det} c_{-a}(i) \neq 0, \quad \operatorname{det} c_{b}(i) \neq 0
$$

for all $a \leqq i \leqq d-b$. It follows directly from the definition of $L$ that

$$
\operatorname{dim}(K=\operatorname{kernel} L)=r(a+b) .
$$

In particular, $f \in K$ is completely determined by

$$
f\left(n_{0}\right), f\left(n_{1}\right), \ldots, f\left(n_{a+b-1}\right) .
$$

For example,

$$
(L f)\left(n_{a}\right)=0
$$


implies

$$
f\left(n_{a+b}\right)=-\left[c_{b}(a)\right]^{-1} \sum_{j=-a}^{b-1} c_{j}(a) f\left(n_{a+j}\right) .
$$

The other values of $f$ are determined inductively.

We define a space $C \subset V$ by

$$
C=\left\{f \in V \mid f\left(n_{i}\right)=0 \quad i<a+b\right\} .
$$

Then

$$
\operatorname{dim} C=r(d+1-(a+b))=\operatorname{dim} K^{\perp}
$$

and

$$
C \cap K=\{0\}
$$

Thus

$$
V=K+C
$$

and we have a corresponding projection

$$
\rho_{C}^{K}: V \rightarrow C .
$$

Let $A$ be any linear subspace of $V$ with

$$
\operatorname{dim} A=r(d+1-(a+b)) .
$$

Then, applying Theorem 1.5 we have, with respect to appropriately chosen volume forms

$$
\operatorname{det} L_{A}=\left(\operatorname{det} \pi_{A^{\perp}} \rho_{K}^{C}: C^{\perp} \rightarrow A^{\perp}\right)(\operatorname{det} L: C \rightarrow \operatorname{Image}(L))\left(\operatorname{det} \pi_{A}: \operatorname{Image}(L) \rightarrow A\right) .
$$

Our goal is to identify the determinants on the right-hand side. We first note that

$$
\text { Image }(L)=\left\{f \in V \mid f\left(n_{i}\right)=0 \text { if } i<a \text { or } i>d-b\right\} \text {. }
$$

Thus, once $A$ is given

$$
\operatorname{det} \pi_{A}: \text { Image }(L) \rightarrow A
$$

can be explicitly calculated.

Let $\left\{e_{1}, \ldots, e_{r}\right\}$ denote the standard basis of $\boldsymbol{C}^{r}$. Define a basis $\left\{f_{i, j}\right\}_{i=0, \ldots, d, j=1, \ldots, r}$ of $V$ by setting for $n_{k} \in N$

$$
f_{i, j}\left(n_{k}\right)= \begin{cases}e_{j} & \text { if } i=k \\ 0 & \text { if } i \neq k .\end{cases}
$$

We define volume forms $\mathscr{V}, \mathscr{L}$ and $\mathscr{C}$, for $V$, Image $(L)$ and $C$, respectively, by

$$
\begin{aligned}
& \mathscr{V}=\left(\bigwedge_{i=1}^{r} f_{0, i}\right) \wedge\left(\bigwedge_{i=1}^{r} f_{1, i}\right) \wedge \cdots \wedge\left(\bigwedge_{i=1}^{r} f_{d, i}\right) \\
& \mathscr{L}=\left(\bigwedge_{i=1}^{r} f_{a, i}\right) \wedge\left(\bigwedge_{i=1}^{r} f_{a+1, i}\right) \wedge \cdots \wedge\left(\bigwedge_{i=1}^{r} f_{d-b, i}\right) \\
& \mathscr{C}=\left(\bigwedge_{i=1}^{r} f_{a+b, i}\right) \wedge\left(\bigwedge_{i=1}^{r} f_{a+b+1, i}\right) \wedge \cdots \wedge\left(\bigwedge_{i=1}^{r} f_{d, i}\right) .
\end{aligned}
$$


Expressing

$$
L: C \rightarrow \operatorname{Image}(L)
$$

as a matrix with respect to the above bases, $L$ is a (block) lower triangle matrix

$$
\left(\begin{array}{cccc}
c_{b}(a) & 0 & 0 \ldots & 0 \\
c_{b-1}(a+1) & c_{b}(a+1) & 0 \ldots & 0 \\
c_{b-2}(a+2) & c_{b-1}(a+2) & c_{b}(a+2) \ldots & 0 \\
\vdots & & & \vdots \\
0 & & & c_{b}(d-b)
\end{array}\right)
$$

This follows by inverting (2.5) to find

$$
L\left(f_{k, l}\right)=\sum_{i=\max \{a, k-b\}}^{\min \{d-b, k+a\}} \sum_{j=1}^{r}\left\langle c_{k-i}(i) e_{l}, e_{j}\right\rangle f_{i, j} .
$$

Thus, with respect to the volume forms $\mathscr{L}$ and $\mathscr{C}$

$$
\operatorname{det}(L: C \rightarrow \operatorname{Image}(L))=\prod_{i=a}^{d-b} \operatorname{det} c_{b}(i)
$$

Lastly, we consider the operator

$$
\pi_{A^{\perp}} \rho_{K}^{C}: C^{\perp} \rightarrow K \rightarrow A^{\perp}
$$

We note that

$$
C^{\perp}=\left\{f \mid f\left(n_{i}\right)=0 \text { for } i \geqq a+b\right\}
$$

and

$$
\rho_{K}^{C}: C^{\perp} \rightarrow K
$$

is the solution of the initial value problem for the finite difference operator $L$. Namely, for $f \in C^{\perp}$

$$
\rho_{K}^{C}(f)=\tilde{f} \in K,
$$

where $\tilde{f} \in K$ is the unique element in the kernel of $L$ such that

$$
\tilde{f}\left(n_{i}\right)=f\left(n_{i}\right) \text { for } i<a+b .
$$

Thus

$$
\rho_{K}^{C}: C^{\perp} \rightarrow K
$$

is the standard identification of an element in the kernel of $L$ with its initial conditions.

A Special Case: Hill's Operator. We now specialize to the case of finite difference approximations to Hill's operator:

$$
\tilde{L}=-\frac{d^{2}}{d x^{2}}+\tilde{R}(x): C^{\infty}\left([0,1], \mathbf{C}^{r}\right) \rightarrow C^{\infty}\left([0,1], \mathbf{C}^{r}\right),
$$

where $\tilde{R}$ is a continuous one-parameter family of $r \times r$ matrices. Fixing $d$, the 
number of nodes, we place the node $n_{i}$ at the point $i / d$ and approximate the operators $d / d x$ and $d^{2} / d x^{2}$ by

$$
\begin{gathered}
\nabla: V \rightarrow V, \\
\nabla^{2}: V \rightarrow V,
\end{gathered}
$$

where

$$
\begin{aligned}
(\nabla f)\left(n_{i}\right) & =d\left[f\left(n_{i+1}\right)-f\left(n_{i}\right)\right] \\
\left(\nabla^{2} f\right)\left(n_{i}\right) & =d\left[(\nabla f)\left(n_{i}\right)-\nabla f\left(n_{i-1}\right)\right] \\
& =d^{2}\left[f\left(n_{i+1}\right)-2 f\left(n_{i}\right)+f\left(n_{i-1}\right)\right] .
\end{aligned}
$$

Set

$$
R\left(n_{i}\right)=\tilde{R}\left(\frac{i}{d}\right)
$$

Then our finite approximation to $\tilde{L}$ is

$$
L=-\nabla^{2}+R: V \rightarrow V .
$$

That is, for $f \in V$,

$$
(L f)\left(n_{i}\right)= \begin{cases}-d^{2} f\left(n_{i-1}\right)+\left[2 d^{2}+R\left(n_{i}\right)\right] f\left(n_{i}\right)-d^{2} f\left(n_{i+1}\right) & \text { if } 0<i<d \\ 0 & \text { if } i=0 \text { or } i=d\end{cases}
$$

or, in the notation of (2.5), $a=b=1$, and for $0<i<d$,

$$
c_{j}(i)= \begin{cases}-d^{2} & \text { if } j= \pm 1 \\ 2 d^{2}+R\left(n_{i}\right) & j=0 \\ 0 & j \neq-1,0,1 .\end{cases}
$$

In this case

$$
\begin{aligned}
C & =\left\{f \in V \mid f\left(n_{0}\right)=f\left(n_{1}\right)=0\right\}, \\
\text { Image }(L) & =\left\{f \in V \mid f\left(n_{0}\right)=f\left(n_{d}\right)=0\right\},
\end{aligned}
$$

and

$$
\operatorname{det} L: C \rightarrow \operatorname{Image}(L)=\prod_{i=1}^{d-1} \operatorname{det} c_{1}(i)=\prod_{i=1}^{d-1}\left(-d^{2}\right)^{r}=\left(-d^{2}\right)^{r(d-1)} .
$$

We now consider different "boundary conditions" $A$.

i) Dirichlet Boundary Conditions. Let

$$
A_{\infty}=\operatorname{Image}(L)=\left\{f \mid f\left(n_{0}\right)=f\left(n_{d}\right)=0\right\} .
$$

This is the so-called Dirichlet boundary condition. (The notation $A_{\infty}$ is chosen to be consistent with the notation of part (ii).) Then

$$
\text { Image }(L) \stackrel{\pi_{A_{\infty}}}{\longrightarrow} A_{\infty}
$$

is the identity, so

$$
\left(\operatorname{det} \pi_{A_{\infty}}: \text { Image }(L) \rightarrow A_{\infty}\right)=1
$$


as long as we take as our volume form $\mathscr{A}_{\infty}$ on $A_{\infty}$ the same form $\mathscr{L}$ that we defined for Image $(L)$. Thus, from Theorem 1.5 and (2.6),

$$
\operatorname{det} L_{A_{\infty}}=\left(-d^{2}\right)^{r(d-1)}\left(\operatorname{det} \pi_{A_{\infty}^{\perp}} \rho_{K}^{C}: C^{\perp} \rightarrow A_{\infty}^{\perp}\right) \text {. }
$$

Now, for simplicity, we set $r=1$. Define a basis $\left\{f_{i}\right\}_{i=0, \ldots, d}$ of $V$ by setting

$$
f_{i}\left(n_{j}\right)= \begin{cases}1 & i=j \\ 0 & i \neq j .\end{cases}
$$

Our volume forms $\mathscr{V}, \mathscr{L}, \mathscr{A}_{\infty}$, and $\mathscr{C}$ of $V$, Image $(L), A_{\infty}$ and $C$, respectively, reduce to

$$
\begin{aligned}
\mathscr{V} & =\bigwedge_{i=0}^{d} f_{i}, \\
\mathscr{A}_{\infty}=\mathscr{L} & =\bigwedge_{i=1}^{d-1} f_{i}, \\
\mathscr{C} & =\bigwedge_{i=2}^{d} f_{i} .
\end{aligned}
$$

Let

$$
\mathscr{C}^{\perp}=f_{0} \wedge f_{1}
$$

be a volume form for $C^{\perp}$, and

$$
\mathscr{A}_{\infty}^{\perp}=(-1)^{d-1} f_{0} \wedge f_{d}
$$

a volume form for $A_{\infty}^{\perp}$.

The sign is chosen so that

$$
\mathscr{C}^{\perp} \wedge \mathscr{C}=\mathscr{A}_{\infty}^{\perp} \wedge \mathscr{A}_{\infty}=\mathscr{V} .
$$

The map

$$
\pi_{A_{\infty}^{\perp}} \rho_{K}^{C}: C^{\perp} \rightarrow A_{\infty}^{\perp}
$$

takes $f_{0} \in C$ to

$$
\left(k_{0}\left(n_{0}\right)\right) f_{0}+\left(k_{0}\left(n_{d}\right)\right) f_{d}=f_{0}+\left(k_{0}\left(n_{d}\right)\right) f_{d},
$$

where $k_{0}=\rho_{K}^{C} f_{0} \in K$ is determined by

$$
k_{0}\left(n_{0}\right)=f_{0}\left(n_{0}\right)=1, \quad k_{0}\left(n_{1}\right)=f_{0}\left(n_{1}\right)=0
$$

and $f_{1}$ to

$$
\left(k_{1}\left(n_{0}\right)\right) f_{0}+\left(k_{1}\left(n_{d}\right)\right) f_{d}=\left(k_{1}\left(n_{d}\right)\right) f_{d},
$$

where $k_{1}=\rho_{K}^{C} f_{1} \in K$ is determined by

$$
k_{1}\left(n_{0}\right)=f_{1}\left(n_{0}\right)=0, \quad k_{1}\left(n_{1}\right)=f_{1}\left(n_{1}\right)=1 .
$$

Thus $\mathscr{C}^{\perp}=f_{0} \wedge f_{1}$ goes to

$$
\begin{aligned}
{\left[f_{0}+k_{0}\left(n_{d}\right) f_{d}\right] \wedge k_{1}\left(n_{d}\right) f_{d} } & =(-1)^{d-1} k_{1}\left(n_{d}\right)\left[(-1)^{d-1} f_{0} \wedge f_{d}\right] \\
& =(-1)^{d-1} k_{1}\left(n_{d}\right) \mathscr{A}_{\infty}^{\perp} .
\end{aligned}
$$


Thus

$$
\left(\operatorname{det} \pi_{A_{\infty}^{\perp}} \rho_{K}^{C}: C \rightarrow A_{\infty}^{\perp}\right)=(-1)^{d-1} k_{1}\left(n_{d}\right) .
$$

Recall that

$$
k_{1}\left(n_{0}\right)=0, \quad k_{1}\left(n_{0}\right)=1
$$

so that

$$
\nabla k_{1}\left(n_{0}\right)=d
$$

Thus

$$
y=\frac{1}{d} k_{1}
$$

satisfies

$$
y\left(n_{0}\right)=0, \quad \nabla y\left(n_{0}\right)=1 .
$$

Combining (2.7) [setting $r=1$ ], (2.8) and (2.9),

\section{Theorem 2.2.}

$$
\operatorname{det} L_{A_{\infty}}=d^{2 d-1} y\left(n_{d}\right) \text {, }
$$

where $y$ is the unique element in the kernel of $L$ satisfying

$$
y\left(n_{0}\right)=0, \quad \nabla y\left(n_{0}\right)=1 .
$$

For $r>1$ the above analysis can be generalized to yield

Theorem 2.3. For general $r$

$$
\operatorname{det} L_{A_{\infty}}=d^{r(2 d-1)} \operatorname{det} Y,
$$

where $Y$ is the $r \times r$ matrix whose $i^{\text {th }}$ column is given by $y_{i}\left(n_{d}\right)$, where $y_{i}$ is the unique element in $K$ such that

$$
y_{i}\left(n_{0}\right)=0, \quad \nabla y_{i}\left(n_{0}\right)=e_{i} .
$$

Observations. 1) Note that as $d \rightarrow \infty$, det $L_{A_{\infty}} \rightarrow \infty$. This is appropriate since, as $d \rightarrow \infty, L_{A_{\infty}}$ approaches

$$
\tilde{L}_{A_{\infty}}=-\frac{d^{2}}{d x^{2}}+\left.\tilde{R}\right|_{\text {Dirichlet }}
$$

which has $\infty$ many eigenvalues which tend to $\infty$.

2) Let

$$
\tilde{L}_{1}=-\frac{d^{2}}{d x^{2}}+\tilde{R}_{1}, \quad \tilde{L}_{2}=-\frac{d^{2}}{d x^{2}}+\tilde{R}_{2}
$$

be Hill's Operators associated to two different potentials. For fixed $d$, let $L_{1}, L_{2}$ be the corresponding finite difference approximations. The relative version of Theorem 2.2 is

$$
\operatorname{det} L_{1, A_{\infty}}\left(L_{2, A_{\infty}}\right)^{-1}=\frac{y_{1}\left(n_{d}\right)}{y_{2}\left(n_{d}\right)}
$$


where $y_{i}$ is the unique element in the kernel of $L_{i}$ satisfying

$$
y_{i}\left(n_{0}\right)=0, \quad \nabla y_{i}\left(n_{0}\right)=1 \text {. }
$$

Taking limits as $d \rightarrow \infty$ we find

$$
\left.\operatorname{det}\left(-\frac{d^{2}}{d x^{2}}+\tilde{R}_{1}(x)\right)\left(-\frac{d^{2}}{d x^{2}}+\tilde{R}_{2}(x)\right)^{-1}\right|_{\text {Dirichlet }}=\frac{\tilde{y}_{1}(x)}{\tilde{y}_{2}(x)}
$$

where $y_{i}$ is the unique solution to

$$
\begin{aligned}
\left(-\frac{d^{2}}{d x^{2}}+\tilde{R}_{i}(x)\right) \tilde{y}_{i}(x) & =0, \\
\tilde{y}_{i}(0)=0 \quad \tilde{y}_{i}^{\prime}(0) & =1,
\end{aligned}
$$

and the left-hand side of (2.11) is a well defined Fredholm (= perturbation) determinant. The technical details required to justify this limiting process are provided in Sect. 3. There is a corresponding formula for general $r$. The formula (2.11) was first proved in [L-S].

3) The operator $L_{A_{\infty}}$ is hermitian, and hence has real eigenvalues.

Taking the sign of both sides of Theorem 2.2, using the fact that $y\left(n_{1}\right)$ is positive, we find

[\# of negative eigenvalues of $\left.L_{A_{\infty}}\right] \equiv$ [\# of sign changes of $y\left(n_{i}\right)$ as $i$ goes from 1 to $d](\bmod 2)$.

This is a weak version of the Morse index theorem [Mi] which states that in the smooth case:

$\left[\#\right.$ of negative eigenvalues of $\left.\tilde{L}_{A_{\infty}}=-\frac{d^{2}}{d x^{2}}+\left.\tilde{R}\right|_{\text {Dirichlet }}\right]=[\#$ of zeros of $\tilde{y}(x)=$ the unique solution of $\tilde{L}$ such that $\left.\tilde{y}(0)=0, \tilde{y}^{\prime}(0)=1\right]$.

4) We can also vary the first order term in $\tilde{L}$. That is, consider

$$
\tilde{L}=-\frac{d^{2}}{d x^{2}}+\tilde{Q}(x) \frac{d}{d x}+\tilde{R}(x)
$$

A complication arises in that there are 3 reasonable ways to approximate $d / d x$ by a finite difference operator, namely by the left, right or symmetric difference. That is

$$
\left[\tilde{Q}(x) \frac{d}{d x} f(x)\right]\left(\frac{i}{d}\right)
$$

can be approximated by

$$
\begin{gathered}
\tilde{Q}\left(\frac{i}{d}\right) \nabla f\left(\frac{i-1}{d}\right), \quad \tilde{Q}\left(\frac{i}{d}\right) \nabla f\left(\frac{i}{d}\right), \quad \text { or } \\
\frac{1}{2} \tilde{Q}\left(\frac{i}{d}\right)\left[\nabla f\left(\frac{i-1}{d}\right)+\nabla f\left(\frac{i}{d}\right)\right],
\end{gathered}
$$

and we will label the resulting finite difference operators $L_{L}, L_{R}$ and $L_{S}$, respectively. In any case, the analysis goes through as before, except possibly for the formula (2.6). 
In the 'first case the formula is unchanged and we learn (in the case of Dirichlet boundary conditions)

$$
\operatorname{det} L_{L}=d^{2 d-1} y_{L}\left(n_{d}\right),
$$

where $y_{L}$ is the unique solution of

$$
L_{L} y_{L}=0, \quad y_{L}(0)=0, \quad \nabla y_{L}(0)=1 .
$$

In the case of $L_{R}$ we have

$$
c_{1}(i)=-d^{2}+Q\left(\frac{i}{d}\right) d
$$

so that (2.6) is replaced by

$$
\begin{aligned}
\operatorname{det} L_{R}: C \rightarrow \operatorname{Im}\left(L_{R}\right) & =\prod_{i=1}^{d-1}\left(-d^{2}+Q\left(\frac{i}{d}\right) d\right) \\
& =(-d)^{2(d-1)} \prod_{i=1}^{d-1}\left(1-\frac{1}{d} Q\left(\frac{i}{d}\right)\right),
\end{aligned}
$$

and we learn

$$
\operatorname{det} L_{R}=d^{2(d-1)}\left[\prod_{i=1}^{d-1}\left(1-\frac{1}{d} Q\left(\frac{i}{d}\right)\right)\right] y_{R}\left(n_{d}\right) .
$$

In the case of $L_{S}$ we have

$$
c_{1}(i)=-d^{2}+\frac{1}{2} \tilde{Q}\left(\frac{i}{d}\right) d
$$

so that (2.6) is replaced by

$$
\begin{aligned}
\operatorname{det} L_{S}: c \rightarrow \operatorname{Im}\left(L_{S}\right) & =\prod_{i=1}^{d-1}\left(-d^{2}+\frac{1}{2} \tilde{Q}\left(\frac{i}{d}\right) d\right) \\
& =\left(-d^{2}\right)^{d-1} \prod_{i=1}^{d-1}\left(1-\frac{1}{2 d} \tilde{Q}\left(\frac{i}{d}\right)\right) .
\end{aligned}
$$

Proceeding as before, we learn

$$
\operatorname{det} L_{S}=d^{2 d-1}\left[\prod_{i=1}^{d-1}\left(1-\frac{1}{2 d} \tilde{Q}\left(\frac{i}{d}\right)\right)\right] y_{S}\left(n_{d}\right)
$$

It is fascinating to note that as $d \rightarrow \infty$,

$$
\begin{aligned}
& \prod_{i=1}^{d-1}\left(1-\frac{1}{d} \tilde{Q}\left(\frac{i}{d}\right)\right) \rightarrow e^{-\int_{0}^{1} \tilde{Q}(x) d x}, \\
& \prod_{i=1}^{d-1}\left(1-\frac{1}{2 d} \tilde{Q}\left(\frac{i}{d}\right)\right) \rightarrow e^{-\frac{1}{2} \int_{0}^{1} \tilde{Q}(x) d x} .
\end{aligned}
$$

In fact, we proved in [Fo1] (Corollary 3.5, suitably interpreted) that there is a $c$ such that

$$
\operatorname{det} \tilde{L}=c e^{-\frac{1}{2} \int_{0}^{1} \tilde{Q}(x) d x} \tilde{y}(1)
$$


(where the left-hand side is defined via zeta function regularization). The constant $c$ is independent of $\widetilde{Q}$ and $\widetilde{R}$. Thus, it appears that $L_{S}$ is the appropriate approximation. However, it is not at all clear how to deduce the above formula for $\tilde{L}$ from the finite difference case. For example, if $\tilde{L}_{1}$ and $\tilde{L}_{2}$ have different first order terms, then $\tilde{L}_{1} \tilde{L}_{2}^{-1}$ does not have a well-defined Fredholm determinant. (The spectrum of $\tilde{L}_{1} \tilde{L}_{2}^{-1}$ looks loosely like $\left\{1+\frac{1}{n} \mid 0 \neq n \in \mathbf{Z}\right\}$ and thus, the product does not converge absolutely). Hence, we cannot use det $\tilde{L}_{1} \tilde{L}_{2}^{-1}$ as we did in Observation 2. However, it follows from the above that, in fact

$$
\frac{\operatorname{det} L_{1, S}^{(d)}}{\operatorname{det} L_{2, S}^{(d)}} \stackrel{d \rightarrow \infty}{\longrightarrow} \frac{\operatorname{det} \tilde{L}_{1}}{\operatorname{det} \tilde{L}_{2}} .
$$

A direct proof of this fact would be very interesting, and may shed some light on the mysterious process of zeta function regularization.

(ii) General Local Boundary Conditions. Fix $\delta_{0}, \delta_{1} \in \mathbf{R}$. In this section we consider the operator $\tilde{L}$ restricted to functions

$$
f:[0,1] \rightarrow \mathbf{C}
$$

which satisfy

$$
f^{\prime}(0)+\delta_{0} f(0)=f^{\prime}(1)+\delta_{1} f(1)=0 .
$$

Note that $\delta_{0}=\delta_{1}=0$ corresponds to the Neumann boundary condition

$$
f^{\prime}(0)=f^{\prime}(1)=0
$$

and, although here we require $\delta_{0}, \delta_{1}<\infty$ the case $\delta_{0}=\delta_{1}=\infty$ corresponds to the Dirichlet boundary condition considered in (i). As before, after fixing $d$, the number of nodes, $\tilde{L}$ is replaced by $L$, and the boundary condition is replaced by

$$
\nabla f\left(n_{0}\right)+\delta_{0} f\left(n_{0}\right)=\nabla f\left(n_{d-1}\right)+\delta_{1} f\left(n_{d}\right)=0,
$$

i.e.

$$
f\left(n_{1}\right)+\left(\frac{\delta_{0}}{d}-1\right) f\left(n_{0}\right)=f\left(n_{d-1}\right)+\left(-\frac{\delta_{1}}{d}-1\right) f\left(n_{d}\right)=0 .
$$

Thus, we define

$$
A_{\delta_{0}, \delta_{1}}=\{f \in V \mid f \text { satisfies }(2.12)\} .
$$

Note that an orthonormal basis for $A_{\delta_{0}, \delta_{1}}$ is provided by

$$
\frac{1}{\alpha_{0}}\left(-f_{0}+\left(\frac{\delta_{0}}{d}-1\right) f_{1}\right), f_{2}, \ldots, f_{d-2}, \frac{1}{\alpha_{1}}\left(f_{d}+\left(\frac{\delta_{1}}{d}+1\right) f_{d-1}\right),
$$

where

$$
\begin{aligned}
& \alpha_{0}=\left|-f_{0}+\left(\frac{\delta_{0}}{d}-1\right) f_{1}\right|=\sqrt{1+\left(\frac{\delta_{0}}{d}-1\right)^{2}}, \\
& \alpha_{1}=\left|f_{d}+\left(\frac{\delta_{1}}{d}+1\right) f_{d-1}\right|=\sqrt{1+\left(\frac{\delta_{1}}{d}+1\right)^{2}} .
\end{aligned}
$$


We define a volume from $\mathscr{A}_{\delta_{0}, \delta_{1}}$ for $A_{\delta_{0}, \delta_{1}}$ by setting

$$
\begin{aligned}
\mathscr{A}_{\delta_{0}, \delta_{1}}= & \frac{1}{\alpha_{0} \alpha_{1}}\left(-f_{0}+\left(\frac{\delta_{0}}{d}-1\right) f_{1}\right) \wedge f_{2} \wedge \cdots \wedge f_{d-2} \\
& \wedge\left(f_{d}+\left(\frac{\delta_{1}}{d}+1\right) f_{d-1}\right) .
\end{aligned}
$$

To find the determinant of the map

$$
\pi_{A}: \text { Image }(L) \rightarrow A
$$

we note that

$$
\begin{aligned}
\pi_{A}\left(f_{i}\right)=f_{i} \quad 2 \leqq i \leqq d-2, \\
\pi_{A}\left(f_{1}\right)=\left\langle f_{1}, \frac{1}{\alpha_{0}}\left(-f_{0}+\left(\frac{\delta_{0}}{d}-1\right) f_{1}\right)\right\rangle \frac{1}{\alpha_{0}}\left(-f_{0}+\left(\frac{\delta_{0}}{d}-1\right) f_{1}\right) \\
=\frac{1}{\alpha_{0}}\left(\frac{\delta_{0}}{d}-1\right)\left[\frac{1}{\alpha_{0}}\left(-f_{0}+\left(\frac{\delta_{0}}{d}-1\right) f_{1}\right)\right], \\
\pi_{A}\left(f_{d-1}\right)=\left\langle f_{d-1}, \frac{1}{\alpha_{1}}\left(f_{d}+\left(\frac{\delta_{1}}{d}+1\right) f_{d-1}\right)\right\rangle \frac{1}{\alpha_{1}}\left(f_{d}+\left(\frac{\delta_{1}}{d}+1\right) f_{d-1}\right) \\
=\frac{1}{\alpha_{1}}\left(\frac{\delta_{1}}{d}+1\right)\left[\frac{1}{\alpha_{1}}\left(f_{d}+\left(\frac{\delta_{1}}{d}+1\right) f_{d-1}\right)\right] .
\end{aligned}
$$

Thus

$$
\operatorname{det}\left(\pi_{A}: \text { Image }(L) \rightarrow A\right)=\frac{1}{\alpha_{0} \alpha_{1}}\left(\frac{\delta_{0}}{d}-1\right)\left(\frac{\delta_{1}}{d}+1\right) .
$$

Combining Theorem 1.5, (2.6) and (2.13),

$$
\operatorname{det} L_{A_{\delta_{0}, \delta_{1}}}=\frac{\left(-d^{2}\right)^{d-1}}{\alpha_{0} \alpha_{1}}\left(\frac{\delta_{0}}{d}-1\right)\left(\frac{\delta_{1}}{d}+1\right) \cdot\left(\operatorname{det} \pi_{A_{\delta_{0}, \delta_{1}}^{\perp}} \rho_{K}^{C}: C^{\perp} \rightarrow A_{\delta_{0}, \delta_{1}}^{\perp}\right) .
$$

Now

$$
A_{\delta_{0}, \delta_{1}}^{\perp}=\operatorname{span}\left\{\left(\left(\frac{\delta_{0}}{d}-1\right) f_{0}+f_{1}\right),\left(\left(\frac{\delta_{1}}{d}+1\right) f_{d}-f_{d-1}\right)\right\} .
$$

We define a volume form $\mathscr{A} \frac{1}{\delta_{0}, \delta_{1}}$ by

$$
\mathscr{A}_{\delta_{0}, \delta_{1}}^{\perp}=\frac{(-1)^{d-1}}{\alpha_{0} \alpha_{1}}\left(\left(\frac{\delta_{0}}{d}-1\right) f_{0}+f_{1}\right) \wedge\left(\left(\frac{\delta_{1}}{d}+1\right) f_{d}-f_{d-1}\right) .
$$

Again, the sign is chosen so that

$$
\mathscr{A}_{\delta_{0}, \delta_{1}}^{\perp} \wedge \mathscr{A}_{\delta_{0}, \delta_{1}}=\mathscr{C}^{\perp} \wedge \mathscr{C}=\mathscr{V}=f_{0} \wedge f_{1} \wedge \cdots \wedge f_{d} .
$$

The map

$$
\pi_{A_{\delta_{0}, \delta_{1}}^{\perp}} \rho_{K}^{C}: C^{\perp} \rightarrow A_{\delta_{0}, \delta_{1}}^{\perp}
$$


takes $f_{0} \in C^{\perp}$ to

$$
\begin{aligned}
& \frac{1}{\alpha_{0}^{2}}\left\langle k_{0},\left(\frac{\delta_{0}}{d}-1\right) f_{0}+f_{1}\right\rangle\left(\left(\frac{\delta_{0}}{d}-1\right) f_{0}+f_{1}\right) \\
& +\frac{1}{\alpha_{1}^{2}}\left\langle k_{0},\left(\frac{\delta_{1}}{d}+1\right) f_{d}-f_{d-1}\right\rangle\left(\left(\frac{\delta_{1}}{d}+1\right) f_{d}-f_{d-1}\right),
\end{aligned}
$$

where $k_{0}$ is the unique element in $K$ satisfying

$$
k_{0}\left(n_{0}\right)=1, \quad k_{0}\left(n_{1}\right)=0,
$$

and $f_{1} \in C^{\perp}$ to

$$
\begin{aligned}
& \frac{1}{\alpha_{0}^{2}}\left\langle k_{1},\left(\frac{\delta_{0}}{d}-1\right) f_{0}+f_{1}\right\rangle\left(\left(\frac{\delta_{0}}{d}-1\right) f_{0}+f_{1}\right) \\
& +\frac{1}{\alpha_{1}^{2}}\left\langle k_{1},\left(\frac{\delta_{1}}{d}+1\right) f_{d}-f_{d-1}\right\rangle\left(\left(\frac{\delta_{1}}{d}+1\right) f_{d}-f_{d-1}\right),
\end{aligned}
$$

where $k_{1} \in K$ is the unique element satisfying

$$
k_{1}\left(n_{0}\right)=0, \quad k_{1}\left(n_{1}\right)=1 \text {. }
$$

Thus $f_{0} \wedge f_{1}$ goes to

$$
\begin{aligned}
& \frac{(-1)^{d-1}}{\alpha_{0} \alpha_{1}}\left[\left\langle k_{0},\left(\frac{\delta_{0}}{d}-1\right) f_{0}+f_{1}\right\rangle\left\langle k_{1},\left(\frac{\delta_{1}}{d}+1\right) f_{d}-f_{d-1}\right\rangle\right. \\
& \left.-\left\langle k_{1},\left(\frac{\delta_{0}}{d}-1\right) f_{0}+f_{1}\right\rangle\left\langle k_{0},\left(\frac{\delta_{1}}{d}+1\right) f_{d}-f_{d-1}\right\rangle\right] \mathscr{A}_{\frac{\delta_{0}}{1} \delta_{1}} .
\end{aligned}
$$

Define $z \in K$ by

$$
z=\left\langle k_{0},\left(\frac{\delta_{0}}{d}-1\right) f_{0}+f_{1}\right\rangle k_{1}-\left\langle k_{1},\left(\frac{\delta_{1}}{d}+1\right) f_{0}+f_{1}\right\rangle k_{0}=\left(\frac{\delta_{0}}{d}-1\right) k_{1}-k_{0} .
$$

Then $z \in K$ is the unique element in $K$ satisfying

$$
z\left(n_{0}\right)=-1, \quad z\left(n_{1}\right)=\frac{\delta_{0}}{d}-1
$$

or, equivalently

$$
z\left(n_{0}\right)=-1, \quad \nabla z\left(n_{0}\right)=\delta_{0} .
$$

Moreover, from (2.17),

$$
\begin{aligned}
\operatorname{det} \pi_{A_{\delta_{0}, \delta_{1}}^{\perp}} \rho_{K}^{C}: C^{\perp} \rightarrow A_{\delta_{0}, \delta_{1}}^{\perp} & =\frac{(-1)^{d-1}}{\alpha_{0} \alpha_{1}}\left\langle z,\left(\frac{\delta_{1}}{d}+1\right) f_{d}-f_{d-1}\right\rangle \\
& =\frac{(-1)^{d-1}}{\alpha_{0} \alpha_{1}}\left(\left(\frac{\delta_{1}}{d}+1\right) z\left(n_{d}\right)-z\left(n_{d-1}\right)\right) \\
& =\frac{(-1)^{d-1}}{d \alpha_{0} \alpha_{1}}\left(\left(\delta_{1}+d\right) z\left(n_{d}\right)-d z\left(n_{d-1}\right)\right) \\
& =\frac{(-1)^{d-1}}{d \alpha_{0} \alpha_{1}}\left(\delta_{1} z\left(n_{d}\right)+\nabla z\left(n_{d-1}\right)\right) .
\end{aligned}
$$

Combining (2.14) and (2.18) yields 


\section{Theorem 2.4.}

$$
\operatorname{det} L_{A_{\delta_{0}, \delta_{1}}}=\frac{d^{2 d-3}}{\alpha_{0}^{2} \alpha_{1}^{2}}\left(\frac{\delta_{0}}{d}-1\right)\left(\frac{\delta_{1}}{d}+1\right)\left(\nabla z\left(n_{d-1}\right)+\delta_{1} z\left(n_{d}\right)\right),
$$

where

$$
\alpha_{0}^{2}=\left(\frac{\delta_{0}}{d}-1\right)^{2}+1, \quad \alpha_{1}^{2}=\left(\frac{\delta_{1}}{d}+1\right)^{2}+1
$$

and $z \in K$ is the unique element in the kernel of $L$ satisfying

$$
z\left(n_{0}\right)=-1, \quad \nabla z\left(n_{0}\right)=\delta_{0} .
$$

Recall that $A_{0,0}$ denotes the Neumann boundary conditions. Theorem 2.4 provides the formula

\section{Corollary 2.5.}

$$
\operatorname{det} L_{A_{0,0}}=\frac{d^{2 d-3}}{4} \nabla z\left(n_{d-1}\right)
$$

where $z \in K$ satisfies

$$
z\left(n_{0}\right)=1, \quad \nabla z\left(n_{0}\right)=0
$$

If

$$
\tilde{L}_{1}=-\frac{d^{2}}{d x^{2}}+\tilde{R}_{1}(x), \quad \tilde{L}_{2}=-\frac{d^{2}}{d x^{2}}+\tilde{R}_{2}(x)
$$

are two operators, then the relative version of Theorem 2.4 yields, upon taking the limit $d \rightarrow \infty$,

$$
\left.\operatorname{det}\left(-\frac{d^{2}}{d x^{2}}+\tilde{R}_{1}(x)\right)\left(-\frac{d^{2}}{d x^{2}}+\tilde{R}_{2}(x)\right)^{-1}\right|_{\tilde{A}_{\delta_{0}, \delta_{1}}}=\frac{\tilde{z}_{1}^{\prime}(1)-\delta_{1} \tilde{z}_{1}(1)}{\tilde{z}_{2}^{\prime}(1)-\delta_{1} \tilde{z}_{2}(1)},
$$

where

$$
\tilde{A}_{\delta_{0}, \delta_{1}}=\left\{f:[0,1] \rightarrow \mathbf{C} \mid f^{\prime}(0)+\delta_{0} f(0)=f^{\prime}(1)+\delta_{1} f(1)=0\right\}
$$

and $\tilde{z}_{i}, i=1,2$, is the unique solution to $\tilde{L}_{i}$ such that

$$
\tilde{z}_{i}(0)=-1, \quad \tilde{z}_{i}^{\prime}(0)=\delta_{0} .
$$

iii) Periodic Boundary Conditions. In this section we consider the operator $\tilde{L}$ acting on functions on the circle $S^{1}$. We associate $S^{1}$ with $[0,1]$ under the identification $0=1$. Then, $C^{1}$ functions on $S^{1}$ can be identified with functions on $[0,1]$ satisfying

$$
f(0)=f(1), \quad f^{\prime}(0)=f^{\prime}(1) .
$$

We think of a finite approximation of $S^{1}$ as given by the interval with $d+1$ evenly spaced nodes under the identification

$$
n_{0}=n_{d-1}, \quad n_{1}=n_{d} .
$$

Then, $C^{1}$ functions on $S^{1}$ are approximated by functions $f \in V$ satisfying

$$
f\left(n_{0}\right)=f\left(n_{d-1}\right), \quad \nabla f\left(n_{0}\right)=\nabla f\left(n_{d-1}\right)
$$


or, equivalently,

$$
f\left(n_{0}\right)=f\left(n_{d-1}\right), \quad f\left(n_{1}\right)=f\left(n_{d}\right) .
$$

Fixing $\delta \in \mathbf{C}$, we generalize (2.18) to the boundary condition

$$
B_{\delta}=\left\{f \in V \mid f\left(n_{0}\right)=\delta f\left(n_{d-1}\right), f\left(n_{1}\right)=\delta f\left(n_{d}\right)\right\} .
$$

An orthonormal basis for $B_{\delta}$ is provided by

$$
\frac{1}{\sqrt{1+|\delta|^{2}}}\left(\delta f_{0}+f_{d-1}\right), \frac{1}{\sqrt{1+|\delta|^{2}}}\left(\delta f_{1}+f_{d}\right), f_{2}, f_{3}, \ldots, f_{d-2} .
$$

Define a volume form $\mathscr{B}_{\delta}$ by

$$
\mathscr{B}_{\delta}=\frac{1}{1+|\delta|^{2}}\left(\delta f_{0}+f_{d-1}\right) \wedge\left(\delta f_{1}+f_{d}\right) \wedge f_{2} \wedge \cdots \wedge f_{d-2} .
$$

To find the determinant of the map

$$
\pi_{B_{\delta}}: \text { Image }(L) \rightarrow B_{\delta}
$$

we note that

$$
\begin{aligned}
& \pi_{B_{\delta}}\left(f_{i}\right)=f_{i} \quad 2 \leqq i \leqq d-2, \\
& \pi_{B_{\delta}}\left(f_{i}\right)=\left\langle f_{1}, \frac{1}{\sqrt{1+|\delta|^{2}}}\left(\delta f_{1}+f_{d}\right)\right\rangle \frac{1}{\sqrt{1+|\delta|^{2}}}\left(\delta f_{1}+f_{d}\right) \\
&=\frac{\delta}{1+|\delta|^{2}}\left(\delta f_{1}+f_{d}\right), \\
& \pi_{B_{\delta}}\left(f_{d-1}\right)=\left\langle f_{d-1}, \frac{1}{\sqrt{1+|\delta|^{2}}}\left(\delta f_{0}+f_{d-1}\right)\right\rangle \frac{1}{\sqrt{1+|\delta|^{2}}}\left(\delta f_{0}+f_{d-1}\right) \\
&=\frac{1}{1+|\delta|^{2}}\left(\delta f_{0}+f_{d-1}\right) .
\end{aligned}
$$

Thus

$$
\mathscr{L}=f_{1} \wedge f_{2} \wedge \cdots \wedge f_{d-1}
$$

goes to

$$
\begin{aligned}
\frac{\delta}{\left(1+|\delta|^{2}\right)^{2}}\left(\left(\delta f_{1}+f_{d}\right) \wedge f_{2} \wedge \cdots \wedge f_{d-2}\right. & \left.\wedge\left(\delta f_{0}+f_{d-1}\right)\right) \\
= & (-1)^{d-2} \frac{\delta}{1+|\delta|^{2}} \mathscr{B}_{\delta} .
\end{aligned}
$$

Combining Theorem 1.5, (2.6) and (2.19),

$$
\operatorname{det} L_{B_{\delta}}=\frac{-d^{2 d-2} \delta}{1+|\delta|^{2}}\left(\operatorname{det} \pi_{B_{\delta}^{\perp}} \rho_{K}^{C}: C^{\perp} \rightarrow B_{\delta}^{\perp}\right) .
$$

Now

$$
B_{\delta}^{\perp}=\operatorname{span}\left\{\left(f_{1}-\delta f_{d}\right),\left(f_{0}-\delta f_{d-1}\right)\right\}
$$


We define a volume form $\mathscr{B}_{\delta}^{\perp}$ by

$$
\mathscr{B}_{\delta}^{\perp}=\frac{1}{1+|\delta|^{2}}\left(f_{0}-\delta f_{d-1}\right) \wedge\left(f_{1}-\delta f_{d}\right)
$$

so that

$$
\mathscr{B}_{\delta}^{\perp} \wedge \mathscr{B}_{\delta}=\mathscr{V}=f_{0} \wedge \cdots \wedge f_{d} .
$$

The map

$$
\pi_{B_{\delta}^{\perp}} \rho_{K}^{C}: C^{\perp} \rightarrow B_{\delta}^{\perp}
$$

takes $f_{0}$ to

$$
\frac{1}{1+|\delta|^{2}}\left\langle k_{0}, f_{0}-\delta f_{d-1}\right\rangle\left(f_{0}-\delta f_{d-1}\right)+\frac{1}{1+|\delta|^{2}}\left\langle k_{0}, f-\delta f_{d}\right\rangle\left(f_{1}-\delta f_{d}\right),
$$

where $k_{0} \in K$ satisfies (2.15), and $f_{2}$ to

$$
\frac{1}{1+|\delta|^{2}}\left\langle k_{1}, f_{0}-\delta f_{d-1}\right\rangle\left(f_{0}-\delta f_{d-1}\right)+\frac{1}{1+|\delta|^{2}}\left\langle k_{1}, f_{1}-\delta f_{d}\right\rangle\left(f_{1}-\delta f_{d}\right),
$$

where $k_{1} \in K$ satisfies (2.16). Thus $\mathscr{C}^{\perp}=f_{0} \wedge f_{1}$ goes to

$$
\frac{1}{1+|\delta|^{2}}\left[\left\langle k_{0}, f_{0}-\delta f_{d-1}\right\rangle\left\langle k_{1}, f_{1}-\delta f_{d}\right\rangle-\left\langle k_{1}, f_{0}-\delta f_{d-1}\right\rangle\left\langle k_{0}, f_{1}-\delta f_{d}\right\rangle\right] \mathscr{B} \frac{\perp}{\delta}
$$

so that

$$
\begin{aligned}
\operatorname{det} & \left(\pi_{B_{\delta}^{\perp}} \rho_{K}^{C}: C^{\perp} \rightarrow B_{\delta}^{\perp}\right) \\
= & \frac{1}{1+|\delta|^{2}}\left[\left\langle k_{0}, f_{0}-\delta f_{d-1}\right\rangle\left\langle k_{1}, f_{1}-\delta f_{d}\right\rangle-\left\langle k_{1}, f_{0}-\delta f_{d-1}\right\rangle\left\langle k_{0}, f_{1}-\delta f_{d}\right\rangle\right] \\
= & \frac{1}{1+|\delta|^{2}}\left[\left(1-\delta k_{0}\left(n_{d-1}\right)\right)\left(1-\delta k_{1}\left(n_{d}\right)\right)+\left(\delta k_{1}\left(n_{d-1}\right)\right)\left(-\delta k_{0}\left(n_{d}\right)\right)\right] \\
= & \frac{1}{1+|\delta|^{2}} \operatorname{det}\left[\left(\begin{array}{ll}
1 & 0 \\
0 & 1
\end{array}\right)-\delta\left(\begin{array}{cc}
k_{0}\left(n_{d-1}\right) & k_{1}\left(n_{d-1}\right) \\
k_{0}\left(n_{d}\right) & k_{1}\left(n_{d}\right)
\end{array}\right)\right] .
\end{aligned}
$$

Thus, from (2.20) and (2.21), we have

Theorem 2.6.

$$
\operatorname{det} L_{B_{\delta}}=\frac{-d^{2 d-2} \delta}{\left(1+|\delta|^{2}\right)^{2}} \operatorname{det}\left[\left(\begin{array}{ll}
1 & 0 \\
0 & 1
\end{array}\right)-\delta\left(\begin{array}{cc}
k_{0}\left(n_{d-1}\right) & k_{1}\left(n_{d-1}\right) \\
k_{0}\left(n_{d}\right) & k_{1}\left(n_{d}\right)
\end{array}\right)\right] .
$$

Unfortunately, the formula (2.22) is not convenient for taking limits as $d \rightarrow \infty$, but (2.22) is easily seen to be equivalent to

$$
\operatorname{det} L_{B_{\delta}}=\frac{-d^{2 d-2} \delta}{\left(1+|\delta|^{2}\right)^{2}} \operatorname{det}\left[\left(\begin{array}{ll}
1 & 0 \\
0 & 1
\end{array}\right)-\delta\left(\begin{array}{cc}
z\left(n_{d-1}\right) & y\left(n_{d-1}\right) \\
\nabla z\left(n_{d-1}\right) & \nabla y\left(n_{d-1}\right)
\end{array}\right)\right] \text {, }
$$

where $z=k_{0}+k_{1}, y=\frac{1}{d} k_{1} \in K$ satisfy

$$
\begin{aligned}
& z\left(n_{0}\right)=1, \quad \nabla z\left(n_{0}\right)=0, \\
& y\left(n_{0}\right)=0, \quad \nabla y\left(n_{0}\right)=1 .
\end{aligned}
$$


If

$$
\tilde{L}_{1}=-\frac{d^{2}}{d x^{2}}+\tilde{R}_{1}(x), \quad \tilde{L}_{2}=-\frac{d^{2}}{d x^{2}}+\tilde{R}_{2}(x)
$$

are two operators, then the relative version of (2.23) leads, upon letting $d \rightarrow \infty$, to

$$
\left.\operatorname{det}\left(-\frac{d^{2}}{d x^{2}}+\tilde{R}_{1}(x)\right)\left(-\frac{d^{2}}{d x^{2}}+\tilde{R}_{2}(x)\right)^{-1}\right|_{B_{\delta}}=\frac{\operatorname{det}\left(\begin{array}{ll}
1 & 0 \\
0 & 1
\end{array}\right)-\delta\left(\begin{array}{ll}
\tilde{z}_{1}(1) & \tilde{y}_{1}(1) \\
\tilde{z}_{1}^{\prime}(1) & \tilde{y}_{1}^{\prime}(1)
\end{array}\right)}{\operatorname{det}\left(\begin{array}{ll}
1 & 0 \\
0 & 1
\end{array}\right)-\delta\left(\begin{array}{ll}
\tilde{z}_{2}(1) & \tilde{y}_{2}(1) \\
\tilde{z}_{2}^{\prime}(1) & \tilde{y}_{2}^{\prime}(1)
\end{array}\right)}
$$

where $\tilde{z}_{i}, \tilde{y}_{i}$ are the unique solutions to $\tilde{L}_{i}$ on $[0,1]$ satisfying

$$
\begin{array}{ll}
\tilde{z}_{i}(0)=1, & \tilde{z}_{i}^{\prime}(0)=0, \\
\tilde{y}_{i}(0)=0, & \tilde{y}_{i}^{\prime}(0)=1 .
\end{array}
$$

\section{The Convergence as $d \rightarrow \infty$}

In this section we prove that as $d \rightarrow \infty$ the formula (2.10) converges to the formula (2.11). More generally, we prove that for any boundary condition, the relevant data from the finite difference operators converges, as the approximation becomes finer, to the corresponding data for the limiting differential operator. Thus, each formula in Sect. 2 for the determinant of a finite difference boundary value operator yields, upon taking limits, a corresponding formula for the determinant of a differential boundary value operator. Our analysis involves two steps. For the right-hand side we must prove the convergence of solutions to the initial value problem. This type of result is standard, but a proof is included for completeness. For the left-hand side, we must prove the convergence of the ratio of determinants. The author has been unable to locate the desired results in the literature, and thus we include a complete proof. Although the results in this section hold for any ordinary differential operator, for simplicity, we restrict attention to $2^{\text {nd }}$ order operators of the type considered in Sect. 2.

Convergence of Solutions of the Initial Value Problem. The right-hand side of (2.10) is expressed in terms of the boundary values at $x=1$ of solutions with prescribed boundary values at $x=0$. Fix $a, b \in \mathbf{C}$. Let $L$ denote the differential operator

$$
L=-\frac{d^{2}}{d x^{2}}+R(x),
$$

where

$$
R:[0,1] \rightarrow \mathbf{C}
$$

is continuous, and let $f$ denote the unique solution to

$$
L f=0, \quad f(0)=a, \quad f^{\prime}(0)=b .
$$

Let $L^{(d)}$ denote the finite difference approximation to $L$ corresponding to 
a division of $[0,1]$ into $d$ equal subintervals, and let $f^{(d)}$ denote the unique solution to

$$
L^{(d)} f^{(d)}=0, \quad f^{(d)}(0)=a, \quad \nabla f^{(d)}(0)=b .
$$

Theorem 3.1. As $d \rightarrow \infty$

$$
\begin{aligned}
f^{(d)}(1) & \rightarrow f(1), \\
\nabla f^{(d)}\left(\frac{d-1}{d}\right) & \rightarrow f^{\prime}(1) .
\end{aligned}
$$

Proof. Let

$$
\varepsilon^{(d)}\left(\frac{i}{d}\right)=f\left(\frac{i}{d}\right)-f^{(d)}\left(\frac{i}{d}\right) .
$$

Then (3.3) is equivalent to

$$
\lim _{d \rightarrow \infty} \varepsilon^{(d)}(1)=0 .
$$

From (3.1) we learn that $f$ is twice continuously differentiable. Thus, in particular,

$$
f^{\prime}(1)=d\left[f(1)-f\left(\frac{d-1}{d}\right)\right]+O\left(\frac{1}{d}\right) .
$$

Since

$$
\nabla f^{(d)}\left(\frac{d-1}{d}\right)=d\left[f^{(d)}(1)-f^{(d)}\left(\frac{d-1}{d}\right)\right],
$$

(3.4) is equivalent to

$$
\lim _{d \rightarrow \infty} d\left[e^{(d)}(1)-e^{(d)}\left(\frac{d-1}{d}\right)\right]=0 .
$$

From Taylor's theorem, for any $x \in(0,1)$,

$$
\begin{aligned}
& f(x-h)=f(x)-f^{\prime}(x) h+\frac{1}{2} f^{\prime \prime}(x) h^{2}+o\left(h^{2}\right), \\
& f(x+h)=f(x)+f^{\prime}(x) h+\frac{1}{2} f^{\prime \prime}(x) h^{2}+o\left(h^{2}\right) .
\end{aligned}
$$

Adding these two equations yields

$$
h^{2} f^{\prime \prime}(x)=-2 f(x)+f(x+h)+f(x-h)+o\left(h^{2}\right) .
$$

Setting $x=i / d$ and $h=1 / d$, we find that for $0<i<d$,

$$
\left(2+\frac{R\left(\frac{i}{d}\right)}{d^{2}}\right) f\left(\frac{i}{d}\right)-f\left(\frac{i+1}{d}\right)-f\left(\frac{i-1}{d}\right)+o\left(\frac{1}{d^{2}}\right)=0 .
$$


On the other hand

$$
\left(2+\frac{R\left(\frac{i}{d}\right)}{d^{2}}\right) f^{(d)}\left(\frac{i}{d}\right)-f^{(d)}\left(\frac{i+1}{d}\right)-f^{(d)}\left(\frac{i-1}{d}\right)=0 .
$$

Therefore

$$
\left(2+\frac{R\left(\frac{i}{d}\right)}{d^{2}}\right) \varepsilon^{(d)}\left(\frac{i}{d}\right)-\varepsilon^{(d)}\left(\frac{i+1}{d}\right)-\varepsilon^{(d)}\left(\frac{i-1}{d}\right)+o\left(\frac{1}{d^{2}}\right)=0 .
$$

Now we examine $\varepsilon$ 's initial conditions. From (3.1) and (3.2),

$$
\varepsilon^{(d)}(0)=0
$$

and

$$
\begin{aligned}
b & =\nabla f^{(d)}(0)=d\left(f^{(d)}\left(\frac{1}{d}\right)-f(0)\right)=d f^{(d)}\left(\frac{1}{d}\right)-d a \\
& =f^{\prime}(0)=d\left(f\left(\frac{1}{d}\right)-f(0)\right)+O\left(\frac{1}{d}\right)=d f\left(\frac{1}{d}\right)-d a+O\left(\frac{1}{d}\right)
\end{aligned}
$$

so that

$$
\varepsilon^{(d)}\left(\frac{1}{d}\right)=O\left(\frac{1}{d^{2}}\right) .
$$

Choose $M$ large enough so that

$$
\begin{aligned}
\left|\varepsilon^{(d)}\left(\frac{1}{d}\right)\right| & \leqq \frac{M}{d^{2}} \text { for all } d, \\
|R(x)| & \leqq M \quad \text { for all } x \in[0,1] .
\end{aligned}
$$

Let $\alpha(d) / d^{2}$ be an upper bound for the norm of the $o\left(1 / d^{2}\right)$ term in (3.8), so that $\lim _{d \rightarrow \infty} \alpha(d)=0$.

We will see that for all $d$, and all $i$ such that $0<i \leqq d$,

$$
\begin{gathered}
\left|\varepsilon^{(d)}\left(\frac{i}{d}\right)\right| \leqq \frac{M}{d} e^{\sqrt{M} \frac{i}{d}}+\frac{\alpha}{d} i\left(1+\frac{M+1}{d}\right)^{i}, \\
\left|\varepsilon^{(d)}\left(\frac{i}{d}\right)-\varepsilon^{(d)}\left(\frac{i-1}{d}\right)\right| \leqq \frac{M}{d}\left(e^{\sqrt{M} \frac{i}{d}}-e^{\sqrt{M} \frac{i-1}{d}}\right)+\frac{\alpha}{d}\left(1+\frac{M+1}{d}\right)^{i} .
\end{gathered}
$$

Setting $i=d$, and using

$$
\lim _{d \rightarrow \infty}\left(1+\frac{M+1}{d}\right)^{d}=e^{M+1}<\infty,
$$


we see that (3.12) implies (3.5), and (3.13) implies (3.6). We will prove (3.12) and (3.13) by induction. From (3.9) and (3.10) both bounds are true for $i=1$. Using (3.8) we can write

$$
\varepsilon^{(d)}\left(\frac{i+1}{d}\right)-\varepsilon^{(d)}\left(\frac{i}{d}\right)=\frac{R\left(\frac{i}{d}\right)}{d^{2}} \varepsilon^{(d)}(i)+\left(\varepsilon^{(d)}\left(\frac{i}{d}\right)-\varepsilon^{(d)}\left(\frac{i-1}{d}\right)\right)+o\left(\frac{1}{d^{2}}\right) .
$$

Thus, by induction and (3.11),

$$
\begin{aligned}
\left|\varepsilon^{(d)}\left(\frac{i+1}{d}\right)-\varepsilon^{(d)}\left(\frac{i}{d}\right)\right| \leqq\left\{\frac{M}{d^{2}}\left(\frac{M}{d} e^{\sqrt{M} \frac{i}{d}}\right)+\frac{M}{d}\left(e^{\sqrt{M} \frac{i}{d}}-e^{\sqrt{M} \frac{i-1}{d}}\right)\right\} \\
+\left\{\frac{M}{d^{2}}\left(\frac{\alpha}{d} i\left(1+\frac{M+1}{d}\right)^{i}\right)+\frac{\alpha}{d}\left(1+\frac{M+1}{d}\right)^{i}+\frac{\alpha}{d^{2}}\right\}=I+I I .
\end{aligned}
$$

Analysing the two terms separately,

$$
\begin{aligned}
I & =\frac{M}{d} e^{\sqrt{M} \frac{i}{d}}\left(1+\frac{M}{d^{2}}-e^{-\sqrt{M} \frac{1}{d}}\right) \leqq \frac{M}{d} e^{\sqrt{M}} \frac{i}{d}\left(e^{\sqrt{M} \frac{1}{d}}-1\right) \\
& =\frac{M}{d}\left(e^{\sqrt{M} \frac{i+1}{d}}-e^{\sqrt{M} \frac{i}{d}}\right) .
\end{aligned}
$$

Using that $\frac{i}{d} \leqq 1$ we have

$$
\begin{aligned}
I I & =\frac{\alpha}{d}\left(\frac{M i}{d^{2}}\left(1+\frac{M+1}{d}\right)^{i}+\left(1+\frac{M+1}{d}\right)^{i}+\frac{1}{d}\right) \\
& \leqq \frac{\alpha}{d}\left(\left(1+\frac{M}{d}\right)\left(1+\frac{M+1}{d}\right)^{i}+\frac{1}{d}\left(1+\frac{M+1}{d}\right)^{i}\right) \\
& =\frac{\alpha}{d}\left(1+\frac{M+1}{d}\right)^{i+1} .
\end{aligned}
$$

These inequalities combine to yield a proof of (3.13). The inequality (3.12) easily follows from (3.8) and the inductive hypothesis.

Convergence of Determinants. Let $R_{0}$ and $R_{1}$ be continuous functions on $[0,1]$, and

$$
\begin{aligned}
& L_{0}=-\frac{d^{2}}{d x^{2}}+R_{0}(x), \\
& L_{1}=-\frac{d^{2}}{d x^{2}}+R_{1}(x)
\end{aligned}
$$

two differential operators acting on functions on $[0,1]$. Let $A$ be a boundary condition given by

$$
\begin{aligned}
& c_{11} f(0)+c_{12} f^{\prime}(0)+c_{13} f(1)+c_{14} f^{\prime}(1)=0, \\
& c_{21} f(0)+c_{22} f^{\prime}(0)+c_{23} f(1)+c_{24} f^{\prime}(1)=0,
\end{aligned}
$$


where

$$
\left(\begin{array}{llll}
c_{11} & c_{12} & c_{13} & c_{14} \\
c_{21} & c_{22} & c_{23} & c_{24}
\end{array}\right)
$$

has rank 2 .

Let $L_{0}^{(d)}$ and $L_{1}^{(d)}$ denote the finite difference approximations of $L_{0}$ and $L_{1}$ corresponding to the partition of $[0,1]$ into $d$ equal subintervals. Approximate $A$ by the finite difference boundary condition

$$
\begin{aligned}
& c_{11} f(0)+c_{12} \nabla f(0)+c_{13} f(1)+c_{14} \nabla f\left(\frac{d-1}{d}\right)=0, \\
& c_{21} f(0)+c_{22} \nabla f(0)+c_{23} f(1)+c_{24} \nabla f\left(\frac{d-1}{d}\right)=0 .
\end{aligned}
$$

We will also denote the approximation by $A$. We will prove that

$$
\lim _{d \rightarrow \infty} \frac{\operatorname{det} L_{1, A}^{(d)}}{\operatorname{det} L_{0, A}^{(d)}}=\operatorname{det} L_{1, A}\left(L_{0, A}\right)^{-1} .
$$

To describe our method of proof, for $\mu \in \mathbf{C}$, let

$$
L_{\mu}=-\frac{d^{2}}{d x^{2}}+R_{0}(x)+\mu\left(R_{1}(x)-R_{0}(x)\right)
$$

and define

$$
\begin{aligned}
D(\mu) & =\operatorname{det} L_{\mu, A}\left(L_{0, A}\right)^{-1}, \\
D^{(d)}(\mu) & =\frac{\operatorname{det} L_{\mu, A}^{(d)}}{\operatorname{det} L_{0, A}^{(d)}} .
\end{aligned}
$$

Note that for each $d$

$$
D(0)=D^{(d)}(0)=1 .
$$

The function $D(\mu)$ is holomorphic (see [G-K]), and non-trivial, by (3.17), and thus has isolated zeros. On the other hand, for each $d, D^{(d)}(\mu)$ is a polynomial, and hence is holomorphic. The analysis of Sect. 2 shows that $D^{(d)}(\mu)$ can be expressed in terms of the boundary values of the solutions to the initial value problem for $L^{(d)}(\mu)$. Theorem 3.1 implies that as $d \rightarrow \infty$, the boundary values, and hence $D^{(d)}(\mu)$, converge uniformly on compact sets in the $\mu$-plane. Thus

$$
\lim _{d \rightarrow \infty} D^{(d)}(\mu)
$$

is holomorphic, and, by (3.17), is non-trivial. Therefore, the zeros of (3.18) are discrete. We prove (3.16) by proving that for each $\mu$ such that

$$
D(\mu) \neq 0 \neq \lim _{d \rightarrow \infty} D^{(d)}(\mu)
$$

we have the equality

$$
\frac{d}{d \mu} \log D(\mu)=\frac{d}{d \mu} \log \lim _{d \rightarrow \infty} D^{(d)}(\mu)
$$


We first examine the left-hand side of (3.19),

$$
\frac{d}{d \mu} \log D(\mu)=\operatorname{tr}\left(\frac{d}{d \mu} L_{\mu, A}\right)\left(L_{\mu, A}\right)^{-1}=\operatorname{tr}\left(R_{1}(x)-R_{0}(x)\right)\left(L_{\mu, A}\right)^{-1}
$$

(see $[\mathrm{G}-\mathrm{K}]$ ). The operator $\left(L_{\mu, A}\right)^{-1}$ has a continuous kernel $G_{\mu, A}(x, y)$, such that for any continuous function $f \in A$,

$$
\left(L_{\mu, A}\right)^{-1} f=\int_{0}^{1} G_{\mu, A}(x, y) f(y) d y .
$$

Equivalently, $G_{\mu, A}(x, y)$ is uniquely determined by the following 4 properties:

(i) Fix $y \in(0,1)$. Then, as a function of $x$,

$$
G_{\mu, A}(x, y) \in A \text {. }
$$

(ii) For $x \neq y$

$$
L_{\mu} G_{\mu, A}(x, y)=0
$$

(where $L_{\mu}$ acts on $G$ as a function of $x$ ).

(iii) The kernel $G_{\mu, A}(x, y)$ is continuous on $[0,1] \times[0,1]$.

(iv) For all $y \in(0,1)$,

$$
\lim _{x \rightarrow y^{-}} \frac{d}{d x} G_{\mu, A}(x, y)=\lim _{x \rightarrow y^{+}} \frac{d}{d x} G_{\mu, A}(x, y)+1 .
$$

(Note that the kernel $G$ is Lipschitz, which implies $L_{\mu}^{-1}$ is a nuclear operator ([G-K]) and thus the discussion in $[\mathrm{G}-\mathrm{K}]$, Chap. IV, applies.)

Therefore,

$$
\left(R_{1}(x)-R_{0}(x)\right)\left(L_{\mu, A}\right)^{-1}
$$

has the continuous kernel

$$
\left(R_{1}(x)-R_{0}(x)\right) G_{\mu, A}(x, y)
$$

with trace

$$
\begin{aligned}
\int_{0}^{1}\left(R_{1}(x)-R_{0}(x)\right) & G_{\mu, A}(x, y) d x \\
= & \lim _{d \rightarrow \infty} \frac{1}{d-2} \sum_{i=1}^{d-1}\left(R_{1}\left(\frac{i}{d}\right)-R_{0}\left(\frac{i}{d}\right)\right) G_{\mu, A}\left(\frac{i}{d}, \frac{i}{d}\right) .
\end{aligned}
$$

Now we consider the right-hand side of (3.19). The uniform convergence of $D^{(d)}(\mu)$ implies

$$
\begin{aligned}
\frac{d}{d \mu} \log \left(\lim _{d \rightarrow \infty} D^{(d)}(\mu)\right) & =\lim _{d \rightarrow \infty}\left(\frac{d}{d \mu} \log D^{(d)}(\mu)\right) \\
& =\lim _{d \rightarrow \infty} \operatorname{tr}\left(\frac{d}{d \mu} L_{\mu, A}^{(d)}\right)\left(L_{\mu, A}^{(d)}\right)^{-1} .
\end{aligned}
$$

The operator $\left(L_{\mu, A}^{(d)}\right)^{-1}$ can be expressed as a matrix

$$
M^{(d)}\left(\frac{i}{d}, \frac{i}{d}\right), \quad 0 \leqq i, j \leqq d
$$


This matrix is uniquely determined by the conditions:

(i) For every $v \in A$,

$$
M v \in A \quad \text { and } \quad L_{\mu, A}^{(d)} M v=v .
$$

(ii) For every $v \in A^{\perp}$,

$$
M v=0 .
$$

Now

$$
\frac{d}{d \mu} L_{\mu, A}^{(d)}=\frac{d}{d \mu} \pi_{A} L_{\mu}^{(d)}=\pi_{A}\left(R_{1}-R_{0}\right),
$$

where $R_{1}-R_{0}$ is the diagonal matrix with

$$
\left(R_{1}-R_{0}\right)\left(\frac{i}{d}, \frac{i}{d}\right)= \begin{cases}0 & i=0 \text { or } d \\ R_{1}\left(\frac{i}{d}\right)-R_{0}\left(\frac{i}{d}\right) & 1 \leqq i \leqq d-1 .\end{cases}
$$

Lastly, we consider the matrix representing $\pi_{A}$. Let

$$
\left(\begin{array}{c}
f(0) \\
\nabla f(0) \\
f(1) \\
\nabla f\left(\frac{d-1}{d}\right)
\end{array}\right)=\operatorname{span}\left\{\left(\begin{array}{l}
a_{1} \\
a_{2} \\
a_{3} \\
a_{4}
\end{array}\right),\left(\begin{array}{l}
b_{1} \\
b_{2} \\
b_{3} \\
b_{4}
\end{array}\right)\right\}
$$

denote the solution space to (3.15). Then (3.24) is equivalent to

$$
\begin{aligned}
\left(\begin{array}{c}
f(0) \\
f\left(\frac{1}{d}\right) \\
f(1) \\
f\left(\frac{d-1}{d}\right)
\end{array}\right) & \left.=\operatorname{span}\left\{\left(\begin{array}{c}
a_{1} \\
a_{1}+\frac{a_{2}}{d} \\
a_{3} \\
a_{3}-\frac{a_{4}}{d}
\end{array}\right), \begin{array}{c}
b_{1} \\
b_{1}+\frac{b_{2}}{d} \\
b_{3} \\
b_{3}-\frac{b_{4}}{d}
\end{array}\right)\right\} \\
& =\operatorname{span}\left\{\left(\begin{array}{l}
a_{1} \\
a_{1} \\
a_{3} \\
a_{3}
\end{array}\right)+\left(\begin{array}{l}
b_{1} \\
b_{1} \\
b_{3} \\
b_{3}
\end{array}\right)\right\}+O\left(\frac{1}{d}\right) \\
& =\operatorname{span}\left\{\left(\begin{array}{l}
1 \\
1 \\
0 \\
0
\end{array}\right),\left(\begin{array}{l}
0 \\
0 \\
1 \\
1
\end{array}\right)\right\}+O\left(\frac{1}{d}\right)
\end{aligned}
$$


as long a's

$$
\operatorname{det}\left(\begin{array}{ll}
a_{1} & b_{1} \\
a_{3} & b_{3}
\end{array}\right) \neq 0 .
$$

We will now assume this. It is not true, the necessary modifications in the argument are clear, and, in fact, lead to some simplification.

Thus, from (3.25),

$$
\pi_{A}=\left(\begin{array}{ccc}
\frac{1}{2} \frac{1}{2}+O\left(\frac{1}{d}\right) & 0 & O\left(\frac{1}{d}\right) \\
0 & I & 0 \\
O\left(\frac{1}{2}\right) & 0 & \frac{1}{2} \frac{1}{2} \frac{1}{2}+O\left(\frac{1}{d}\right)
\end{array}\right) .
$$

Combining (3.22), (3.23) and (3.26) we see

$$
\begin{aligned}
\operatorname{tr}\left(\frac{d}{d \mu} L_{\mu, A}^{(d)}\right)\left(L_{\mu, A}^{(d)}\right)^{-1} & =\sum_{i=1}^{d-1}\left(R_{1}\left(\frac{1}{d}\right)-R_{0}\left(\frac{1}{d}\right)\right) M^{(d)}\left(\frac{i}{d}, \frac{i}{d}\right) \\
& +\sum_{(i, j) \in S} O\left(\frac{1}{d}\right) M\left(\frac{i}{d}, \frac{i}{d}\right),
\end{aligned}
$$

where

$$
S=\{(1,0),(1,1),(1, d-1),(1, d),(d-1,0),(d-1,1),(d-1, d-1),(d-1, d)\} .
$$

Comparing (3.20) to (3.27) we see that the desired equality (3.19) follows from the two lemmas:

Lemma 3.2. There is a constant $c$ (independent of $d$ ) such that for $i, j \in$ $\{0,1, d-1, d\}$,

$$
\left|M^{(d)}\left(\frac{i}{d}, \frac{i}{d}\right)\right| \leqq c .
$$

Lemma 3.3. a) For $j=1$, and $d-1$,

$$
\left|M\left(\frac{i}{d}, \frac{i}{d}\right)-\frac{1}{d} G\left(\frac{i}{d}, \frac{i}{d}\right)\right|=O\left(\frac{1}{d}\right) .
$$

b) For $2 \leqq j \leqq d-2$

$$
\left|M\left(\frac{i}{d}, \frac{i}{d}\right)-\frac{1}{d} G\left(\frac{i}{d}, \frac{i}{d}\right)\right|=o\left(\frac{1}{d}\right),
$$

where the above bounds are uniform in $i$ and $d$.

We will see that Lemmas 3.2 and 3.3 reduce to

Lemma 3.4. If $L_{A}$ is invertible, then there is a constant $c>0$ (independent of $d$ ) such that for all d large enough

$$
\left\|L_{A}^{(d)} v\right\|_{\infty} \geqq c\|v\|_{\infty} \quad \text { for all } v \in A
$$

(where $\left.\|v\|_{\infty}=\sup _{0 \leqq i \leqq d}\left|v\left(\frac{i}{d}\right)\right|\right)$. 
We assume Lemma 3.4 for the moment, and prove Lemmas 3.2 and 3.3. Proof of Lemma 3.2. From (3.25) there are two vectors in $A$ of the form

$$
v_{1}=\left(\begin{array}{c}
1 \\
1 \\
0 \\
0 \\
\vdots \\
0
\end{array}\right)+O\left(\frac{1}{d}\right) \quad v_{2}=\left(\begin{array}{c}
0 \\
\vdots \\
0 \\
0 \\
1 \\
1
\end{array}\right)+O\left(\frac{1}{d}\right)
$$

and thus $A^{\perp}$ is spanned by two vectors of the form

$$
v_{3}=\left(\begin{array}{c}
1 \\
-1 \\
0 \\
0 \\
\vdots \\
0
\end{array}\right)+O\left(\frac{1}{d}\right) \quad v_{4}=\left(\begin{array}{r}
0 \\
\vdots \\
0 \\
0 \\
1 \\
-1
\end{array}\right)+O\left(\frac{1}{d}\right)
$$

Thus, from the definition of $M^{(d)}$,

$$
\begin{aligned}
& M^{(d)} v_{1} \in A, \quad L_{\mu, A}^{(d)}\left(M^{(d)} v_{1}\right)=v_{1}, \\
& M^{(d)} v_{2} \in A, \quad L_{\mu, A}^{(d)}\left(M^{(d)} v_{2}\right)=v_{2}, \\
& M^{(d)} v_{3}=0, \\
& M^{(d)} v_{4}=0 .
\end{aligned}
$$

From (3.28) and Lemma 3.4, there is a $c$ such that

$$
\left\|M v_{1}\right\|_{\infty},\left\|M v_{2}\right\|_{\infty} \leqq c .
$$

Thus we have the following bound (uniformly in $i$ and $d$ )

$$
\left(\begin{array}{c}
M v_{1}\left(\frac{i}{d}\right) \\
M v_{2}\left(\frac{i}{d}\right) \\
M v_{3}\left(\frac{i}{d}\right) \\
M v_{4}\left(\frac{i}{d}\right)
\end{array}\right)=O(1)
$$


From (3.28) and (3.29)

$$
\left.\left(\begin{array}{c}
M v_{1}(i) \\
M v_{2}\left(\frac{i}{d}\right) \\
M v_{3}\left(\frac{i}{d}\right) \\
M v_{4}\left(\frac{i}{d}\right)
\end{array}\right)=\left[\begin{array}{rrrr}
1 & 1 & 0 & 0 \\
0 & 0 & 1 & 1 \\
1 & -1 & 0 & 0 \\
0 & 0 & 1 & -1
\end{array}\right)+O\left(\frac{1}{d}\right)\right]\left(\begin{array}{c}
M\left(\frac{i}{d}, 0\right) \\
M\left(\frac{i}{d}, \frac{1}{d}\right) \\
M\left(\frac{i}{d}, \frac{d-1}{d}\right) \\
M\left(\frac{i}{d}, 1\right)
\end{array}\right),
$$

which shows that

as desired.

$$
\left(\begin{array}{c}
M\left(\frac{i}{d}, 0\right) \\
M\left(\frac{i}{d}, \frac{1}{d}\right) \\
M\left(\frac{i}{d}, \frac{d-1}{d}\right) \\
M\left(\frac{i}{d}, 1\right)
\end{array}\right)=O(1)
$$

Proof of Lemma 3.3. a) We consider the case $j=1$. Let

$$
\tilde{M}\left(i, \frac{1}{d}\right)=\frac{1}{2}\left(M v_{1}-M v_{3}\right) \text {. }
$$

From (3.28) and (3.29),

$$
\tilde{M}\left(i, \frac{1}{d}\right)=M\left(i, \frac{1}{d}\right)+O\left(\frac{1}{d}\right)
$$

so it is enough to prove

$$
\left|\tilde{M}\left(i, \frac{1}{d}\right)-\frac{1}{d} G\left(i, \frac{1}{d}\right)\right|=O\left(\frac{1}{d}\right) .
$$

From (3.30),

$$
\tilde{M}\left(i, \frac{1}{d}\right) \in A, \quad L_{\mu, A}^{(d)}\left(\tilde{M}\left(i, \frac{1}{d}\right)\right)=\frac{1}{2} v_{1} .
$$

We know $G\left(x, \frac{1}{d}\right) \in A$ (as a function of $x$ ). That is,

$$
\left(G\left(0, \frac{1}{d}\right),\left.\frac{d}{d x}\right|_{x=0} G\left(x, \frac{1}{d}\right), G\left(1, \frac{1}{d}\right),\left.\frac{d}{d x}\right|_{x=1} G\left(x, \frac{1}{d}\right)\right)
$$


satisfies (3.14). In addition

$$
\begin{aligned}
& \left.\frac{d}{d x}\right|_{x=0} G\left(x, \frac{1}{d}\right)=\nabla_{1}^{(d)} G\left(0, \frac{1}{d}\right)+O\left(\frac{1}{d}\right), \\
& \left.\frac{d}{d x}\right|_{x=1} G\left(x, \frac{1}{d}\right)=\nabla_{1}^{(d)} G\left(\frac{d-1}{d}, \frac{1}{d}\right)+O\left(\frac{1}{d}\right)
\end{aligned}
$$

(where the subscript 1 denotes the difference is taken with respect to the first variable). Thus

$$
\left(G\left(0, \frac{1}{d}\right), \nabla_{1}^{(d)} G\left(0, \frac{1}{d}\right), G\left(1, \frac{1}{d}\right), \nabla_{1}^{(d)} G\left(\frac{d-1}{d}, \frac{1}{d}\right)\right) \in A+O\left(\frac{1}{d}\right) .
$$

Moreover, as in (3.7), for $2 \leqq i \leqq d-1$,

$$
O=-G^{\prime \prime}\left(\frac{i}{d}, \frac{1}{d}\right)+R\left(\frac{i}{d}\right) G\left(\frac{i}{d}, \frac{1}{d}\right)=L_{\mu, A}^{(d)} G\left(\frac{i}{d}, \frac{1}{d}\right)+o(1) .
$$

From Taylor series

$$
G\left(\frac{2}{d}, \frac{1}{d}\right)=G\left(\frac{1}{d}, \frac{1}{d}\right)+\frac{1}{d} \lim _{x \rightarrow \frac{1}{d}}+\frac{d}{d x} G\left(x, \frac{1}{d}\right)+\frac{1}{2 d^{2}} G^{\prime \prime}\left(z_{1}, \frac{1}{d}\right)
$$

for some $z_{1} \in\left(\frac{1}{d}, \frac{2}{d}\right)$.

Thus

Similarly

$$
\lim _{x \rightarrow \frac{1}{d}} \frac{d}{d x} G\left(x, \frac{1}{d}\right)=\nabla_{1} G\left(\frac{1}{d}, \frac{1}{d}\right)-\frac{1}{2 d} R\left(z_{1}\right) G\left(z_{1}, \frac{1}{d}\right) .
$$

$$
\lim _{x \rightarrow \frac{1}{d}} \frac{d}{d x} G\left(x, \frac{1}{d}\right)=\nabla_{1} G\left(0, \frac{1}{d}\right)+\frac{1}{2 d} R\left(z_{2}\right) G\left(z_{2}, \frac{1}{d}\right)
$$

for some $z_{2} \in\left(0, \frac{1}{d}\right)$.

Thus

$$
\begin{aligned}
-1 & =\lim _{\substack{1 \\
x \rightarrow \frac{1}{d}}} \frac{d}{d x} G\left(x, \frac{1}{d}\right)-\lim _{\substack{1 \\
x \rightarrow \frac{1}{d}}} \frac{d}{d x} G\left(x, \frac{1}{d}\right) \\
& =\nabla_{1} G\left(\frac{1}{d}, \frac{1}{d}\right)-\nabla_{1} G\left(0, \frac{1}{d}\right)-\frac{1}{2 d}\left[R\left(z_{1}\right) G\left(z_{1}, \frac{1}{d}\right)+R\left(z_{2}\right) G\left(z_{2}, \frac{1}{d}\right)\right] \\
& =\nabla_{1}^{2}\left[\frac{1}{d} G\left(\frac{1}{d}, \frac{1}{d}\right)\right]-R\left(\frac{1}{d}\right)\left[\frac{1}{d} G\left(\frac{1}{d}, \frac{1}{d}\right)\right]+o\left(\frac{1}{d}\right) .
\end{aligned}
$$

(The error is $o(1 / d)$ since both $R$ and $G$ are continuous.)

$$
=-\left.L_{\mu, A}^{(d)} G\left(\frac{i}{d}, \frac{1}{d}\right)\right|_{i=1}+o\left(\frac{1}{d}\right) .
$$


Therefore,

$$
L_{\mu}^{(d)}\left[\frac{1}{d} G\left(\frac{i}{d}, \frac{1}{d}\right)\right]=\left(\begin{array}{c}
0 \\
1 \\
0 \\
\vdots \\
0
\end{array}\right)+o\left(\frac{1}{d}\right)
$$

This implies, using (3.27),

$$
\begin{aligned}
L_{\mu, A}^{(d)} \frac{1}{d} G\left(\frac{i}{d}, \frac{1}{d}\right) & =\pi_{A} L_{\mu}^{(d)}\left[\frac{1}{d} G\left(\frac{i}{d}, \frac{1}{d}\right)\right] \\
& =\left(\begin{array}{c}
\frac{1}{2} \\
\frac{1}{2} \\
0 \\
\vdots \\
0
\end{array}\right)+O\left(\frac{1}{d}\right) .
\end{aligned}
$$

Let

$$
\varepsilon\left(\frac{i}{d}\right)=\tilde{M}\left(\frac{i}{d}, \frac{1}{d}\right)-\frac{1}{d} G\left(\frac{i}{d}, \frac{1}{d}\right) .
$$

Then, from (3.31) and (3.33),

$$
\pi_{A} L_{\mu}^{(d)} \varepsilon=O\left(\frac{1}{d}\right)
$$

and

$$
\left(\varepsilon(0), \nabla \varepsilon(0), \varepsilon(1), \nabla \varepsilon\left(\frac{d-1}{d}\right)\right)+O\left(\frac{1}{d^{2}}\right)
$$

satisfies (3.15).

Let $\tilde{\varepsilon}(i / d)$ denote the unique function which has boundary values equal to the $O\left(1 / d^{2}\right)$ term in (3.34) and is linear in $i$ for $1 \leqq i \leqq d-1$. Then

$$
\begin{aligned}
\tilde{\varepsilon}\left(\frac{i}{d}\right) & =O\left(\frac{1}{d^{2}}\right), \\
L_{\mu}^{(d)} \tilde{\varepsilon} & =O\left(\frac{1}{d}\right) .
\end{aligned}
$$

Thus,

$$
\varepsilon-\tilde{\varepsilon} \in A
$$

and

$$
\pi_{A} L_{\mu}^{(d)}(\varepsilon-\tilde{\varepsilon})=O\left(\frac{1}{d}\right)
$$


From Lemma 3.4

$$
\varepsilon-\tilde{\varepsilon}=O\left(\frac{1}{d}\right)
$$

so that

$$
\varepsilon=O\left(\frac{1}{d}\right)
$$

as desired.

b) The case $2 \leqq j \leqq d-2$ is easier than $j=1, d-1$. Following as above to (3.32) we learn that

$$
\varepsilon\left(\frac{i}{d}\right)=M\left(\frac{i}{d}, \frac{i}{d}\right)-\frac{1}{d} G\left(\frac{i}{d}, \frac{i}{d}\right)
$$

satisfies

$$
L_{\mu}^{(d)} \varepsilon=\left(\begin{array}{c}
0 \\
0 \\
\vdots \\
1 \\
\vdots \\
0
\end{array}\right)+o\left(\frac{1}{d}\right)
$$

so that

$$
\pi_{A} L_{\mu}^{(d)} \varepsilon=\left(\begin{array}{c}
0 \\
0 \\
\vdots \\
1 \\
\vdots \\
0
\end{array}\right)+o\left(\frac{1}{d}\right)
$$

(where the 1 is in the $j^{\text {th }}$ spot). Continuing as in (a) we learn

$$
\varepsilon=o\left(\frac{1}{d}\right)
$$

as desired.

We now complete this section with a proof of Lemma 3.4.

Proof of Lemma 3.4. Suppose the lemma is false. That is, suppose there is a sequence

$$
d_{i} \rightarrow \infty
$$

and

$$
v_{i} \in A^{\left(d_{i}\right)}
$$


such that

$$
\begin{gathered}
\left\|v_{i}\right\|_{\infty}=\sup _{\substack{0 \leqq j \leqq d_{i} \\
\left\|L_{A}^{\left(d_{2}\right)} v_{i}\right\|_{\infty} \rightarrow 0 .}}\left|v_{i}\left(\frac{j}{d_{i}}\right)\right|=1,
\end{gathered}
$$

Extend $L^{\left(d_{i}\right)} v_{i}$ linearly between the nodes $\left\{\frac{j}{d}\right\}$ to a continuous function on $[0,1]$. Then define a sequence of $C^{2}$ functions $\tilde{v}_{i}$ by

$$
\begin{aligned}
L \tilde{v}_{i} & =L^{\left(d_{i}\right)} v_{i}, \\
\tilde{v}_{i}(0) & =v_{i}(0), \\
\tilde{v}_{i}^{\prime}(0) & =\nabla v_{i}(0) .
\end{aligned}
$$

Then a very slight modification of the proof of Theorem 3.1 yields

$$
\begin{aligned}
\tilde{v}_{i}\left(\frac{j}{d_{i}}\right)-v_{i}\left(\frac{j}{d_{i}}\right) & \rightarrow 0, \\
\tilde{v}_{i}^{\prime}\left(\frac{j}{d_{i}}\right)-\nabla v_{i}\left(\frac{j-1}{d_{i}}\right) & \rightarrow 0
\end{aligned}
$$

uniformly in $i$ and $j$. (For this conclusion, we need to know that $v_{i}(0)$ and $\nabla v_{i}(0)$ are bounded uniformly in $i$. The bound on $v_{i}(0)$, as well as a bound on $v_{i}(1)$, follows from (3.35). The bound on $\nabla v_{i}(0)$ follows from the bounds on $v_{i}(0)$ and $v_{i}(1)$, the fact that $v_{i} \in A$, and our assumption (3.25).)

We note that, by definition,

$$
L^{\left(d_{i}\right)} v_{i}(0)=L^{\left(d_{i}\right)} v_{i}(1)=0 .
$$

Thus, as can be seen from the form of $\pi_{A}$ in (3.26), (3.36) implies

$$
\left\|L^{\left(d_{i}\right)} v_{i}\right\|_{\infty} \rightarrow 0
$$

so that, in fact,

$$
\left\|L \tilde{v}_{i}\right\|_{\infty} \rightarrow 0 \text {. }
$$

Since $\tilde{v}_{i}(0)$ and $\tilde{v}_{i}^{\prime}(0)$ are bounded, we can find an $a, b$ and a subsequence of the $\tilde{v}_{i}$ such that

$$
\tilde{v}_{i}(0) \rightarrow a, \quad \tilde{v}_{i}^{\prime}(0) \rightarrow b .
$$

Then (3.38) implies that these $\tilde{v}_{i}$ approach $\tilde{v}$, uniformly in $C^{1}[0,1]$, where $\tilde{v}$ is the unique solution to

$$
L \tilde{v}=0, \quad \tilde{v}(0)=a, \quad \tilde{v}^{\prime}(0)=b .
$$

The uniformity of the convergence implies

$$
\tilde{v}_{i}(1) \rightarrow \tilde{v}(1), \quad \tilde{v}_{1}^{\prime}(1) \rightarrow \tilde{v}^{\prime}(1) .
$$

Comparing with (3.37) we learn

$$
\begin{array}{ll}
v_{i}(0) \rightarrow \tilde{v}(0) & \nabla v_{i}(0) \rightarrow \tilde{v}^{\prime}(0), \\
v_{i}(1) \rightarrow \tilde{v}(1) & \nabla v_{i}\left(\frac{d_{i}-1}{d_{i}}\right) \rightarrow \tilde{v}^{\prime}(1) .
\end{array}
$$


Since each $v_{i} \in A$, we must have $\tilde{v} \in A$.

Lastly, we note that the uniform bound

$$
\begin{aligned}
\left\|\tilde{v}_{i}\right\|_{\infty} & \geqq \sup _{j}\left|\tilde{v}_{i}\left(\frac{j}{d_{i}}\right)\right|=\sup _{j}\left|v_{i}\left(\frac{j}{d_{i}}\right)+o(1)\right| \\
& =1+o(1)
\end{aligned}
$$

implies

$$
\tilde{v} \neq 0 \text {. }
$$

Thus $\tilde{v} \in A$ is a non-trivial solution to $L \tilde{v}=0$, which contradicts the hypothesis of the lemma.

\section{References}

[B-F-K] Burghelea, D., Friedlander, L., Kappeler, T.: On the Determinant of Elliptic Differential and Finite Difference Operators in Vector Bundles over $S^{1}$. Commun. Math. Phys. (1991) (to appear)

[B-D] Bott, R., Duffin, R.: On the Algebra of Networks. Trans. Am. Math. Soc. 74, 99-103 (1953)

[D-D] Dreyfuss, T., Dym, H.: Product formulas for the eigenvalues of a class of boundary value problems. Duke Math. J. 45, 15-37 (1978).

[Fo1] Forman, R.: Functional Determinants and Geometry. Inv. Math. 88, 447-493 (1987)

[Fo2] Forman, R.: Difference Operators, Covering Spaces and Determinants. Topology 28, 413-438 (1989)

[Fo3] Forman, R.: Determinants of Laplacians on Graphs, preprint

[Fr] Franklin, P.: The Electric Currents in a Network. J. Math. Phys. 4, 97-102 (1925)

[G-K] Gohberg, I.C., Krein, M.G.: Introduction to the theory of linear nonselfadjoint operators; Vol. 18 (Translation of Mathematical Monographs). Providence, Rhode Island: American Mathematical Society 1969

[Ki] Kirchhoff, G.: Über die Auflösung der Gleichungen auf welche man beider Untersuchen der Linearen Verteilung galvanischer Ströme geführt wird. Annal. Physik Chemie 72, 495-508 (1847)

[L-S] Levit, S., Smilansky, U.: A theorem on infinite products of eigenvalues of SturmLiouville type operators. Proc. Am. Math. Soc. 65, 299-302 (1977)

[Mi] Milnor, J.: Morse Theory. Annal Math. Stud., Vol. 51. Princeton, NJ: Princeton University Press 1963

[Ra] Ramond, P.: Field Theory, A Modern Primer. Frontiers in Physics, Vol. 51. Reading, MA: Benjamin/Cummings 1981

[R-S] Ray, D.B., Singer, I.M.: R-Torsion and the Laplacian on Riemannian manifolds. Adv. Math. 7, 145-210 (1971)

Communicated by A. Jaffe 